\title{
Approche expérimentale de techniques paléolithiques de façonnage de roches peu aptes à la taille
}

\author{
In: Paléo. N. 5,1993. pp. 155-177.
}

\begin{abstract}
This paper deals with some working techniques of nonflint stones, such as pecking, hammering, polishing with or without an abrasive agent. After having observed archaeological pieces with traces of theses techniques, we carried out experimentations on varying techniques and raw materials in order to evaluate the relative qualities of these different raw materials and the efficiency of the different tested techniques. A comparison with archaeological documents allows to conclude to a differentiated treatment of nonflint stones at the Upper Palaeolithic. Moreover, these experimentations give some insight on the question of the need of an intermediate abrasive agent for polishing operations ; or that of the distinction between traces of polish due to the fabrication and traces of polish due to the use.
\end{abstract}

\section{Résumé}

II sera traité ici de certaines techniques de façonnage de roches peu aptes à la taille, telles que piquetage, martelage, émeulage et abrasion. Après observation de pièces archéologiques présentant des stigmates de ces techniques, nous avons entrepris des expérimentations en faisant varier techniques et matières premières afin d'estimer les qualités relatives de ces différentes matières premières et l'efficacité des différentes techniques testées. Une confrontation avec les documents archéologiques permet de conclure à un traitement différencié des roches autres que le silex au Paléolithique supérieur. En outre, ces expérimentations permettent de soulever une nouvelle fois différentes questions telles que celle de l'efficacité du recours à un agent abrasif intermédiaire lors d'opérations de polissage ; ou encore celle de la différenciation entre traces de polissage de fabrication et traces de poli d'usure.

Citer ce document / Cite this document :

de Beaune Sophie A. Approche expérimentale de techniques paléolithiques de façonnage de roches peu aptes à la taille. In: Paléo. N. 5,1993. pp. 155-177.

doi : 10.3406/pal.1993.1109

http://www.persee.fr/web/revues/home/prescript/article/pal_1145-3370_1993_num_5_1_1109

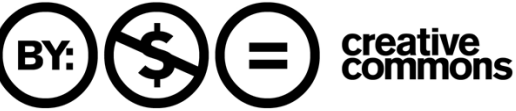




\title{
APPROCHE EXPERIMENTALE DE TECHNIQUES PALEOLITHIQUES DE FAÇONNAGE DE ROCHES PEU APTES À LA TAILLE
}

\author{
Sophie A. de Beaune*
}

\begin{abstract}
Résumé :
Il sera traité ici de certaines techniques de façonnage de roches pcu aptes à la taille, telles que piquetage, martelage, émeulage et abrasion. Après observation de pièces archéologiques présentant des stigmates de ces techniques, nous avons entrepris des expérimentations en faisant varier techniques et matières premières afin d'estimer les qualités relatives de ces différentes matières premières et l'efficacité des différentes techniques testées. Une confrontation avec les documents archéologiques permet de conclure à un traitement différencié des roches autres que le silex au Paléolithique supéricur. En outre, ces expérimentations permettent de soulever une nouvelle fois différentes questions telles que celle de l'efficacité du recours à un agent abrasif intcrmédiaire lors d'opérations de polissage ; ou encore celle de la différenciation entre traces de polissage de fabrication et traces de poli d'usure.
\end{abstract}

\begin{abstract}
:
This paper deals with some working techniques of nonflint stones, such as pecking, hammering, polishing with or without an abrasive agent. After having observed archaeological pieces with traces of theses techuiques, we carried out experimentations on varying techniques and raw materials in order to evaluate the relative qualities of these different raw materials and the efficiency of the different tested techniques. A comparison with archaeological documents allows to conclude to a differentiated treatment of nonflint stones at the Upper Palaeolithic. Moreover, these experimentations give some insight on the question of the need of an intermediate abrasive agent for polishing operations; or that of the distinction between traces of polish due to the fabrication and traces of polish due to the use.
\end{abstract}

Mots-clefs : Paléolithique supćrieur, outils de picrre non taillćs, piquetage, martelage, polissage, abrasion, émeulage, expérimentation.

Key-words : Upper Paleolithic, nunflint stone tools, pecking, hammering, polishing, smoothing, abrasion, experimentation.

Parmi les nombreux vestiges jonchant un sol d'habitat paléolithique, on néglige un peu deux catégories d'objets de pierre, qui ont pourtant contribué à de multiples activités domestiques. Il s'agit d'une part des objets façonnés par une technique autre que la taille, d'autre part des blocs, galets et plaquettes utilisés a l'état brut et portant des traces d'usage. Nous avons traité ailleurs (de Beaune 1989 a ct b, 1992, 1993 ct à paraître) des objets de la seconde catégorie et nous limiterons ici à ceux de la première catégorie. Nous avons aussi exclu de cette étude les galets taillés car ils sont largement étudiés par les spécialistes du lithique (voir, entre autres, MacRae et Moloney 1988 et Sussman 1988).

Les techniques de façonnage considérées ici relèvent de la percussion lancée et de la percussion posée (Leroi-Gourhan 1943, p. 47-64). Pour la percussion lancée, nous considérerons ici le martclage et le piquetage, qui consistent tous deux à mettre une pointe en contact avec la surface travaillée. On parle de piquetage si l'outil utilisé est un pic robustc à bout pointu, de martelage si c'est un gros percutcur à bout arrondi attaquant la matière par écrasement. Le type d'outil utilisé sur du matériel archćologique est assez facile d̀ identifier grâce à la dimension des impacts de percussion.

Pour la percussion posée, nous considérerons le polissage, qui relève de la percussion posée diffuse ${ }^{(1)}$. On peut théoriquement distinguer deux types de polissage : l'abrasion ou l'émeulage ${ }^{(2)}$, selon qu'on utilise ou non un abrasif ; mais pour le matériel archéologique, la distinction est difficile car le recours à un abrasif n'est pas perceptible même à la loupe binoculaire ${ }^{(3)}$. Le terme de polissage ne sera donc utilisé ici que lorsqu'il ne sera pas possible de déterminer si un abrasif a été utilisé ou non. En

1. Nous avons traité ailleurs de la percussion posée linćaire - le raclage. Nous l'avions testée lors de la confection de lampes expérimentales en grès (de Beaune 1987, p. 103-105). Nous menons actucllement des expérimentations du même type, avec Ph. Alix, sur de la stéatite (voir plus loin).

2. Nous utilisons le terme d'émeulage à la suite d'André Prous et de José Garanger : il s'agit “d'une simple abrasion des aspérités, qui n'amène pas la formation d'une surface parfaitement polie, unie et brillante" (Prous 1977, p. 66-67). Pour J. Garanger, auquel A. Prous fait lui-même référence dans le texte cité, l'émeulage se fait "sur polissoir sans abrasif intermédiaire, la présence de celui-ci permettant seul le polissage" (Garanger 1968, p. 18).

3. On a déjà remarqué (Chevalier, Inizan et Tixier 1982, p. 58 et 61) qu'il est difficile de distinguer les stries laissées par des grains de sable abrasif des traces laissées par les grains de quartz du broyeur ou de la molette eux-mêmes, souvent choisis dans une matière première rugueuse pour sa qualité abrasive naturelle. 
revanche, nous avons toujours spécifié le type de polissage utilisé lors de nos expérimentations.

\section{TECHNIQUES PALÉOLITHIQUES DE FAÇONNAGE DE ROCHES PEU APTES A LA TAILLE}

Nous avons déjà observé que bon nombre de lampes et de récipients paléolithiques avaient fait l'objet d'un façonnage intégral, ou d'aménagements partiels tels que cannelures, rigoles ou encore creusement de la cuvette (de Beaune 1987). Les techniques de façonnage utilisées varient selon la nature de la matière première. Ainsi, le grès semble préférentiellement associé à la percussion posée tandis que d'autres roches, telles que le calcaire, sont plutôt travaillées par percussion lancée.

Il est légitime de se demander si d'autres objets présentaient de tels types de travail au Palćolithique supérieur. C'est effectivement le cas d'objets non utilitaires, comme les œuvres d'art mobilier et pariétal, sculptées et non gravées ${ }^{(4)}$. Pour les objets utilitaires, hors les lampes et récipients, la chose est rare. On peut supposer que les Paléolithiques ramassaient de préférence des blocs et galets dont la forme naturelle requérait peu d'aménagement ${ }^{(5)}$. On n'observe le plus souvent, surtout sur les roches dures, que quelques légers aménagements ne modifiant guère la forme générale de l'objet. C'est le cas de certains galets présentant une cannclure aménagće dont l'usage est inconnu mais qui n'ont certainement pas servi de lampe ou de récipient étant donné la petitesse de leur cavité (fig. 1). C'est aussi celui de certains broyeurs et molettes qui présentent, sur une partie de leur surface, des traces de piquetage évoquant les préparations de surface des meules protohistoriques (fig. 2)(6).

Il ne nous a pas paru nécessaire, à ce stade de l'étude, de dresser un inventaire exhaustif des objets façonnés de ce type. Nous avons plutôt dressé un catalogue des techniques attestées, en portant notre choix sur les objets utilitaires, que nous connaissons bien. Quelques pièces du Musée des Antiquités Nationales (M.A.N.) représentent un éventail suffisamment large des techniques d'aménagement et de façonnage utilisées sur diverses roches.

\section{Percussion lancée}

Dès l'Aurignacien, la percussion lancée est utilisée pour le façonnage ou l'aménagement de certaines surfaces - comme l'attestent certains blocs décorés dont les traits profonds ont été obtenus par un piquetage serré (Delluc 1978) - et elle perdure tout au long du Paléolithique supérieur et même audelà. Le piquetage et le martelage ${ }^{(7)}$ sont utilisés notamment pour obtenir des cupules, des cuvettes ou des cannelures. Nous les avons observés sur plusieurs roches différentes, plutôt caractérisées par leur dureté et par un grain moyen ou grossier, souvent irrégulier: en particulier sur du calcaire (fig. 3), sur du granite, très difficile à entamer autrement (fig. 4) et sur certains grès, en particulier ceux à grain grossier (fig. 5).

\section{Percussion posée}

Le polissage, qu'il s'agisse d'émeulage ou d'abrasion, apparaît dès l'Aurignacien, comme l'attestent de nombreuses statuettes humaines et animales. En revanche, les objets utilitaires en pierre polie sont très rares avant le Magdalénien ${ }^{(8)}$. Le polissage scrt soit à un façonnage intégral, soit à la régularisation de surfaces façonnées par d'autres techniques. Dans le premier cas, il s'agit bien souvent de roches tendres, faciles à travailler, comme certains grès à grain fin ou des laves bulleuses (fig. 6). Dans le second cas, le poli tend à occulter les traces de façonnage antérieur et à rendre leur observation difficile. C'est par exemple le cas du brûloir de Lascaux. Il arrive aussi qu'avant d'y graver un décor, on ait préparé une surface par polissage ou raclage (fig. 7). Quand la fonction de l'objet est inconnue, il est difficile de savoir si le poli provient de l'utilisation ou de la fabrication. C'est le cas de la cannelure circulaire du galet de Saint-Marcel, dont le piquctage est partiellement occulté par des traces de poli intense, d'origine inexpliquée (fig. 1).

4. Citons les petits animaux sculptés en ronde-bosse (grotte d'Tsturitz, La Madeleine, Le Mas d'Azil, etc.), les blocs ornés de cupules et de cannelures (abri Reverdit, abri Cellier, etc.), ou les parois rocheuses sculptées (Angles-sur-l'Anglin, Le Roc de Sers, le Cap-Blanc, etc.).

5. M. Ricq-de Bouard constate aussi, en étudiant des haches polies néolithiques, que la phase de débitage est en général absente, la plupart des objets étant fabriqués à parir de galets choisis pour leurs formes et leurs dimensions, et ne nécessitant plus qu'un façonnage, le plus souvent réalisé par piquetage, et une finition (1983, p. 11)

6. C'est ce que les anglo-saxons appellent rejuvenation pitting, procédé de ravivage destiné à redonner sa rugosité première, son "mordant", à la surface, rendue ainsi plus efficace pour moudre ou broyer.

7. Nous avons évité le terme de bouchardage, la boucharde étant inconnue au Palćolithique. L'emploi de termes tels que pierre "pointée, talottée, bouchardée, égrisée, adoucie, polie ..." (Delluc 1978) serait d'ailleurs à redéfinir ou à abandonner. C'est le geste et l'outil qui doivent servir à désigner la technique et non une vague analogie d'aspect de surface.

8. Les plus belles lampes, entièrement sculptées et polics, sont toutes magdaléniennes. Les trois lampes sculptées du Bois du Roc (Charente), qui seraient antérieures au Magdalénien, ont une origine doutcuse ct leur datation serait à revoir. 


\section{Ambiguïté entre traces de façonnage et stigmates d'utilisation}

Avant d'aborder l'expérimentation, rappelons qu'il n'est pas toujours facile de distinguer les traces de fabrication des traces d'utilisation.

Ainsi, des traces de raclage dans un godet à peintures peuvent avoir été faites aussi bien au moment de la confection de l'objet qu'au moment du malaxage des couleurs. Dans ce cas, seule la superposition des différents vestiges peut permettre de conclure. Dans d'autres cas, comme pour le poli d'utilisation visible sur les broyeurs et molettes, c'est la localisation des traces qui permettra de trancher. Lorsque l'utilisation de l'objet est inconnue, même des traces nettes peuvent être difficiles à interpréter. Ainsi, en plus d'un net poli d'utilisation et de traces d'impacts sur ses deux faces planes (provenant d'une utilisation en marteau ou en enclume), une petite molette de grès rose de la grotte d'Isturitz présente sur tout son pourtour des traces de piquetage. Ontelles été obtenues volontairement pour régulariser la forme du galet ou bien fortuitement lors de son utilisation en marteau sur tout son pourtour (fig. 8)(9)? Signalons également les galets martelés baptisés "sphérö̈des" qu'on trouve dans les plus anciennes industries humaines. Willoughby a interprété leurs traces de percussion (pecking) comme des stigmates de fabrication (1987) mais il peut aussi s'agir de traces d'utilisation.

La même ambiguïté se retrouve pour les traces de poli. B. Madsen constate, lors du façonnage expérimental de haches polies en silex, qu'il est difficile de distinguer un poli d'utilisation d'un polissage délibéré $(1984$, p. 56). Certains auteurs estiment que l'orientation des striations pourrait permettre de trancher (Roodenberg 1983) mais cette opinion est controversée (ibid., discussion, p. 187 188). On peut à ce sujet dire un mot du lustrage. Nous n'avons pas observé de roches ayant fait l'objet d'un lustrage de façonnage (à l'aide de plantes siliceuses par exemple) au Palćolithique. Le lustrage observé sur l'extrémité ou les bords des galets généralement appelés "lissoirs" est vraisemblement un lustrage d'utilisation, provenant peut-ĉtre du travail du cuir ou de peaux. Le seul cas ambigu de lustre de fabrication a été observé sur une pièce de Pair-non-Pair, malheureusement mal calée stratigraphiquement mais datant de toute façon du début du Paléolithique supérieur. Ses bords et l'extrémité de ses deux faces ont été polis et lustrés au point qu'ils se rejoignent en formant une arête vive (fig. 9) ${ }^{(10)}$.

\section{TECHNIQUES EXPÉRIMENTALES DE FAÇONNAGE DE ROCHES PEU APTES À LA TAILLE}

Après observation de pièces archéologiques présentant des stigmates de piquetage, martelage, émeulage ou abrasion, nous avons entrepris de comparer expérimentalement l'effet de ces quatre techniques sur trois matières premières différentes. Nous avons donc fait varier techniques et matières premières afin d'estimer les qualités relatives des premières et l'efficacité des secondes. Chaque test consistait à chercher à obtenir, dans un temps d'expérimentation constant, une cuvette la plus régulière et la plus profonde possible. Nous avons délibérément choisi un temps d'expérimentation assez limité : une demie-heure. Une expérimentation de plus longue durée aurait forcément homogénéisé les résultats et rendu leur comparaison plus difficile ; prolongée pendant plusieurs heures, elle aurait en effet produit des cuvettes larges et profondes dans tous les cas.

Les expérimentations ont été suivies de l'observation à la loupe binoculaire Nachet des blocs travaillés et des outils utilisés (de x 10 à $\times 20$ ). Les prises de vue ont été effectuées à un grossissement moindre (jusqu'à x 10).

\section{Les matières premières sélectionnées :}

1) les supports travaillés : trois blocs de matière première différente ont été choisis, chaque technique étant testée à chaque fois dans une partie différente du bloc. Les matières premières ont été choisies parce qu'on les retrouve dans certains artefacts palćolithiques que nous avons eu à étudier. Il s'agit d'un granite jaune de Bretagne (Pointe de la Torche, Finistère) (11), que nous avons choisi très dur comme le sont certains galets de granite palcolithiques creusés d'une cupule (de Beaune 1987) ; d'un calcaire blanc un pcu gréscux à grains serrés, avec quartz (Le Buisson, Dordogne), proche de nombreux récipients et lampes paléolithiques (de Beaune, ibid.) ; et d'un

9. Notons que les Aborigènes australiens utilișent des outils semblables. Tout à la fois molette, meule et enclume, ces petits galets, appelés kulki, ont eu un usage prolongé comme percuteur sur tout leur pourtour, ce qui leur a donné une forme cylindrique. Leur pourtour présente donc une surface régularisée acquise par suite de ce martelage prolongé. Ce qui pourrait passer à première vue pour du façonnage est donc en fait dû à l'usure. Accessoirement, leurs faces planes ont servi à diverses opérations de mouture (McCarthy 1976, p. 59).

10. L'objet évoque un "prototype de hache" et rappelle les ébauchoirs et ciseaux de Bouqras publiés par J. Roodenberg (1983, fig. 1, $\mathrm{n}^{\circ} 5$ et 6) 11. Nous avons également utilisé d'autres granites de Bretagne : deux granites gris (Pointe du Millet et Pointe de la Torche) et un autre rose à grain fin (Ploumanac'h). Les résultats n'étant pas sensiblement différents de ceux obtenus avec le granite jaune, nous n'avons pas jugé utile de les mentionner ici. 
grès rose permien à grain fin (Collonges-la-Rouge, Corrèze), identique à celui choisi pour la confection des plus belles lampes sculptées (de Beaune, ibid.).

2) les outils : plusieurs types ont été utilisés : pour le martelage, des percuteurs en roche éruptive (basalte), ramassés près du village du Buisson, probablement charriés par la Dordogne depuis le Massif central ; pour le piquetage, des pics en silex assez robustes ${ }^{(12)}$; pour l'émeulage et l'abrasion, un galet de gneiss à grain moyen ramassé dans une carrière près de Brive ainsi que des petits fragments de grès de Fontainebleau formé de grains de silice de $0,2 \mathrm{~mm}$ en moyenne, agglomérés par du carbonate de calcium. Là encore, il s'agit de matières premières comparables à celles qui étaient disponibles en Dordogne au Paléolithique et dont on trouve de nombreux fragments dans les habitats ${ }^{(13)}$.

3) l'abrasif : on a utilisé du sable de mer de la côte Atlantique (donc chargé de beaucoup de quartz), imbibé d'eau.

Voici, brièvement décrits, les principaux résultats obtenus sur chaque bloc et pour chaque technique.

\section{Percussion lancée}

\section{Martelage}

Sur le bloc de granite. On utilise un percuteur de basalte à extrémité mousse ; on obtient, par petits coups répétés, une petite cuvette ronde. A la loupe binoculaire, les traces d'impacts disséminées sur la surface apparaissent circulaires dans le fond ct plus allongées sur les versants. Les cristaux de quartz sont "déchaussés" (fig. 10.1). L'extrémité du percuteur est complètement écrasée et esquillée, le bloc de granite étant beaucoup plus dur que le basalte.

Sur le bloc de calcaire. Un autre percuteur de basalte à extrémité mousse est utilisé. Le calcaire ne s'écrase pas, il s'effrite. Le creusement de la cuvette s'effectue en bordure du bloc et entraîne un écaillement du bord. Il apparaît qu'il vaut mieux utiliser un pic de silex pour l'attaquer. A la loupe binoculaire, les traces d'écrasement apparaissent allongées, ovalaires, plus grandes que celles obtenues lors du piquetage. Les versants des creux obtenus sont abrupts et les creux sont séparés par des crêtes pointues (fig. 10.2). Le percuteur est à peine usé.

Sur le bloc de grès rose tendre. Avec un percuteur de basalte, on obtient une belle cuvette à surface un peu irrégulière, plus régulière cependant que la surface naturelle (fig. 10.3). Le percuteur est peu usé mais des grains de sable rose sont visibles dans les anfractuosités de son extrémité. Au bout de dix minutes, une fissure traversant le bloc commence à se former. On continue à marteler tout en surveillant attentivement cette fissure qui va en s'intensifiant. Le bloc résiste mais cette technique apparaît cependant peu propice au travail d'un grès tendre.

\section{Piquetage}

Sur le bloc de granite. La percussion se fait par petits coups répétés. La pointe du pic en silex étant plus fine que celle des percuteurs utilisés pour le martelage, la percussion est plus sélective. On déplace fréquemment le bloc, ce qui permet d'obtenir une petite cupule, plus profonde que celle obtenue par martelage, et bien circulaire. Le pic est assez usé. A la loupe binoculaire, les traces d'impacts sont circulaires, plus ponctuelles que les traces de martelage et plus profondes. On observe aussi des traces de "raclage" correspondant au dérapé du pic par endroits (fig. 10.4). Les cristaux de quartz sont "déchaussés". L'extrémité du pic est écrasée, émoussée.

Sur le bloc de calcaire. Comme pour le granite, le pic apparaît plus sélectif. La cuvette obtenue est bien ronde, plus régulière et plus petite mais plus profonde que celle obtenue par martelage. A la loupe binoculaire, les traces de piquetage apparaissent circulaires. Les creux ont un versant oblique à la différence des dépressions obtenues lors du martelage, a bords abrupts. On voit que les grains les plus durs ont été déchaussés et on observe aussi des stries entre ces grains comme sur le granite (fig. 10.5). Le pic est assez usé, mais moins que le précédent.

Sur le bloc de grès rose tendre. Pour creuser une cuvette régulièrement circulaire, il vaut mieux partir d'un point central et l'élargir. On obtient une cuvette un peu moins régulière mais beaucoup plus profonde et aux bords beaucoup plus abrupts que par martelage. La fissure apparue pendant le martelage ne s'est pas modifiée pendant le piquetage. Comme sur les autres matières premières, on observe quelques traces de "raclage" là où le pic a dérapé (fig. 10.6). L'extrémité du pic est à peine émoussée, on y distingue une légère coloration rose.

\section{Percussion posée}

\section{Emeulage}

Le même galet de gneiss a été utilisé dans les trois cas, toujours sur la même extrémité. Deux petits 
fragments de grès de Fontainebleau ont également été testés sur leurs faces et leurs arêtes.

Sur le bloc de granite. Ni le gneiss, ni le granite ne libérant de cristaux abrasifs, le résultat obtenu est peu convaincant : beaucoup de travail pour peu de résultats. Les aspérités sont bien éliminées mais les creux subsistent. Cette technique apparaît peu adaptée à ce matériau. A la loupe binoculaire, les angles des cristaux apparaissent émoussés ; on observe aussi de fines stries. Les creux naturels de la roche interrompent la surface régularisée (fig. 11.1). En travaillant plusieurs heures d'affilée, on pourrait obtenir une surface entièrement régularisée. En revanche, l'utilisation d'un petit bloc de grès à grain grossier permet d'obtenir rapidement une surface régulière, très légèrement concave. Les grains de silice libérés du petit bloc de grès forment un abrasif naturel.

Sur le bloc de calcaire. On obtient, avec le galet de gneiss, la régularisation d'une surface assez rugueuse au départ, mais ni creusement, ni même aplanissement. A la loupe binoculaire, la surface de la roche est bien régulière. Les cristaux sont émoussés et présentent quelques stries (fig. 11.2). Comme précédemment, aucune des deux matières premières ne libérant de grains abrasifs, l'action du gneiss sur le calcaire est presque nulle. En revanche, le recours à un petit bloc de grès permet d'obtenir rapidement une surface adoucie et légèrement creusée surtout caractérisée par la présence de nombreuses petites stries fines parallèles. Là encore, la libération des grains de silice du grès a joué le même rôle que le recours à un sable abrasif intermédiaire. Après utilisation sur le granite, puis sur le calcaire, toutes les arêtes du petit bloc de grès sont émoussées.

Sur le bloc de grès rose tendre. Avec le galet de gneiss, on obtient une surface bien polie et même légèrement surcreusée. A la loupe binoculaire, cette surface est très régulière et ne présente aucune trace de raclage (fig. 11.3). C'est ici le support travaillé qui a fourni l'abrasif intermédiaire, le galet de gneiss déchaussant les grains de silice du bloc de grès. L'utilisation d'un nouveau petit bloc de grès de Fontainebleau à grain grossier sur le support en grès permien à grain fin donne des résultats intéressants. Tous deux, broyeur et support, libèrent des grains de silice qui se mêlent pour former un puissant abrasif attaquant la surface des deux objets. La surface travaillée du bloc de grès rose est lisse, plane, très légèrement concave, sans stries apparentes. Le petit bloc a ses faces et ses flancs un peu convexes entièrement régularisés, toutes les aspérités ayant disparues. Son aspect rappelle celui de certains broyeurs paléolithiques dont on peut ainsi supposer que la forme régulière a été obtenue lors de l'utilisation et non lors d'une mise en forme antérieure à l'utilisation.

\section{Abrasion}

Le même galet de gneiss que précédemment a été utilisé, mais sur son autre extrémité. Le sable de mer utilisé comme abrasif est imbibé d'eau. On prend garde de ne pas mettre trop de sable abrasif entre le bloc à travailler et l'outil pour éviter le phénomène "d'aquaplanage" observé par Jacques Pelegrin ${ }^{(14)}$.

Sur le bloc de granite. Le résultat est beaucoup plus efficace que celui obtenu lors de l'émeulage. La surface est entièrement régularisée et même légèrement surcreusée. Il ne subsiste pratiquement plus de creux naturels. A la loupe binoculaire, on voit que les cristaux sont entièrement émoussés. Quelques grains de sable subsistent dans certains creux (fig. 11.4).

Sur le bloc de calcaire. Là encore, l'abrasion est plus efficace que l'émeulage. La surface polie est plus étendue que celle obtenue par l'émeulage et elle est même légèrement concave. Les cristaux sont émoussés et les stries visibles à la loupe binoculaire, et même à l'œil nu, ont toutes à peu près la même direction (fig. 11.5). L'aspect de surface obtenu est très proche de celui que l'on observait précédemment après émeulage avec un petit fragment de grès.

Sur le bloc de grès rose tendre. On obtient une large cuvette à bords évasés beaucoup plus étendue et profonde que celle obtenue par l'émeulage. A la loupe binoculaire, la zone abrasée apparaît aussi régulière que la zone régularisée par émeulage (fig. 11.6). On y observe quelques grains de quartz provenant du sable utilisé comme abrasif.

Le galet de gneiss présente, à l'œil nu, peu de traces d'usure. A la loupe binoculaire, l'extrémité utilisée sans abrasif présente des traces de poli et des stries parallèles tandis quc l'autre extrémité présente par endroits un véritable lustre réfléchissant la lumière ; on y observe quelques grains de silice incrustés.

\section{CONCLUSION}

Ces expérimentations apportent tout d'abord des informations au niveau tracéologique. Les surfaces

14. S. Rostain et $Y$. Wack confirment, lors de l'étude de la fabrication de haches et herminettes de Guyane, que le dosage d'eau et de sable est important (1987, p. 123). B. Madsen a également constaté l'efficacité de l'utilisation du sable et de l'eau comme agent abrasif, contrairement à l'emploi de graviers qui produisent moins de friction, créant une sorte d'effet de "roulement à billes" (ball-bearing effect) nuisant à l'efficacité (1984, p. 57). 
travaillées expérimentalement présentent effectivement, à la loupe binoculaire et à l'œil nu, des stigmates de fabrication très variables, ce qui autorise à les comparer aux documents paléolithiques. Notons cependant que, dans le cas du matériel archéologique, seule la dernière opération est visible et que le finissage par polissage ou raclage efface le façonnage antérieur, ce qui est d'ailleurs son but. On ne peut donc identifier, sur les pièces archéologiques, que les techniques utilisées lors du dernier stade de fabrication, sauf si l'on est en présence d'ébauche, de préforme ou d'objet cassé en cours de fabrication. Il peut aussi arriver qu'une pièce n'ait pas été régularisée sur la totalité de sa surface, et des rugosités résiduelles peuvent alors permettre de reconnaître certaines techniques de dégrossissage et de façonnage.

$\mathrm{La}$ différenciation entre martelage et piquetage est assez aisée : il ne s'agit finalement que d'une difference de dimensions des impacts de percussion. Notons cependant que l'on peut obtenir un aspect différent en modifiant la force d'impact mais avec le même outil. On peut ainsi régulariser les cupulettes produites par un "martelage" puissant en frappant à coups moins forts et plus rapprochés (de Beaune, Roussot, Sackett 1986, p. 111). Par ailleurs, nous avons vu plus haut que la différence entre abrasion et émeulage est très difficile à faire archéologiquement. Il n'est d'ailleurs pas sûr que l'expérimentation apporte grand chose de nouveau dans ce domaine. En effet, que l'on utilise une roche non abrasive, comme le gneiss, avec un sable abrasif intermédiaire, ou bien une roche abrasive comme le grès, l'effet produit sur la roche travaillée sera le même et le résultat obtenu très comparable. On peut ajouter à cela qu'une surface lissée et striée visible sur un bloc ou une plaquette de provenance archéologique peut avoir été produite aussi bien lors de son façonnage que lors de son utilisation : l'abrasion d'une surface - avec un abrasif intermédiaire ou avec une roche abrasive donnera le même résultat que la mouture ou l'écrasemont d'une matière quelconque à l'aide d'une molette ou d'un broyeur en grès. Ainsi, certaines meules des grottes de La Vache et d'Isturitz présentent de multiples petites stries fines parallèles sur leurs surfaces usées. C'est surtout la présence de colorant rouge dans ces stries qui permet de considérer ces objets comme des meules à ocre ${ }^{(1)}$ (de Beaune et Buisson, à paraître).

L'observation directe des objets sous le microscope s'est révélée assez décevante, les impuretés de surface gênant la lecture des stigmates. De même, nous avons moulé des échantillons de surface travaillée expérimentalement avec du vernis nitro-cellulosique ou une feuille d'acétate. L'examen au microscope des empreintes n'a rien donné de concluant. Par ailleurs, les traces observées sur le matériel expérimental varient selon la matière première testéc. Il faudrait donc, pour constituer une grille de référence valide, engager un nouveau protocole expérimental pour chaque matière première étudiée. On peut s'interroger sur la rentabilité d'une telle démarche étant donné la grande variabilité des matières premières ramassées sous forme de blocs, de galets et de plaquettes.

L'expérimentation apporte également des informations au niveau technologique, et celles-ci sont plus riches. Pour les deux techniques de percussion lancée, la profondeur et le diamètre des cuvettes obtenues ont été comparés (graph. 1 et 2).

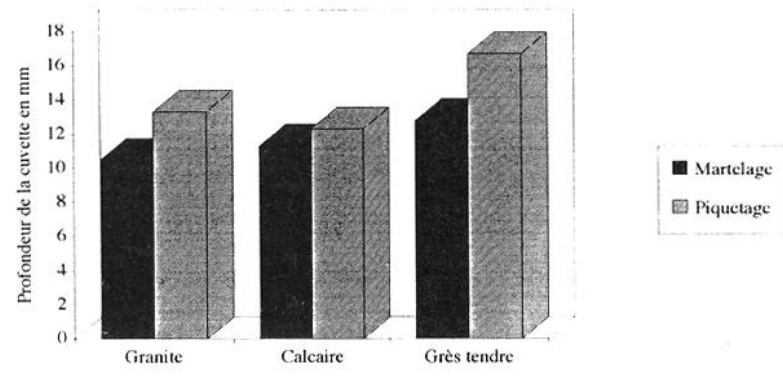

Graphique 1.- Profondeur des cuvettes obtenues expérimentalement lors du martelage avec percuteurs de basalte et du piquetage avec pics en silex (en mm).

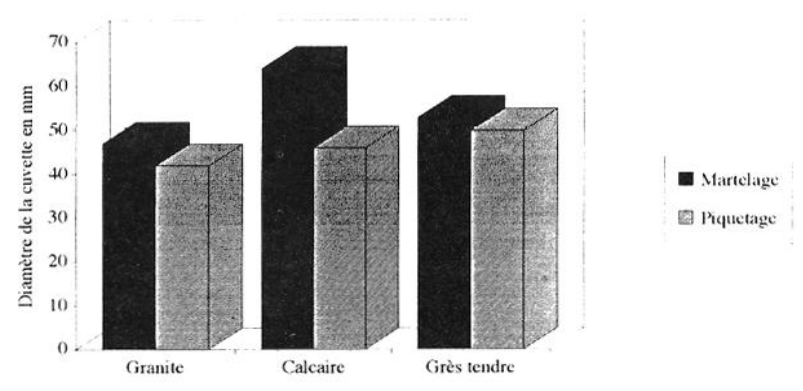

Graphique 2.- Diamètre des cuvettes obtenues expérimentalement lors du martelage avec percuteurs de basalte et du piquetage avec pics en silex (en mm).

15. Mais l'hématite peut aussi être utilisée comme abrasif. Sa présence peut donc indiquer aussi bien la fonction d'un objet que son mode de fabrication. 
On peut en conclure que le martelage permet d'obtenir des cavités larges et peu profondes. Il est donc plus efficace pour un travail de dégrossissage. Par ailleurs, il s'est révélé peu adapté au travail du grès fin car les chocs trop violents risquent d'entraîner la cassure du bloc travaillé. Ceci avait d'ailleurs déjà été constaté lors de précédentes expérimentations, sur des grès plus ou moins compacts (de Beaune 1987, p. 103-109). Le piquetage permet d'obtenir des cavités plus profondes et moins larges que celles obtenues par martelage. C'est donc une technique adaptée à un travail plus précis. Elle s'est averrée efficace sur les trois matières premières testées. On peut l'utiliser sur du grès à condition qu'il soit assez léger pour éviter les risques de cassure, le grès étant plus ou moins solide, selon la taille des grains qui le composent, et surtout selon leur degré de cohésion.

Pour les deux techniques de percussion posée, la qualité du poli obtenu ainsi que la capacité de creusement de la matière ont été comparées (graph. 3 et 4). La qualité du poli a été provisoirement classće,

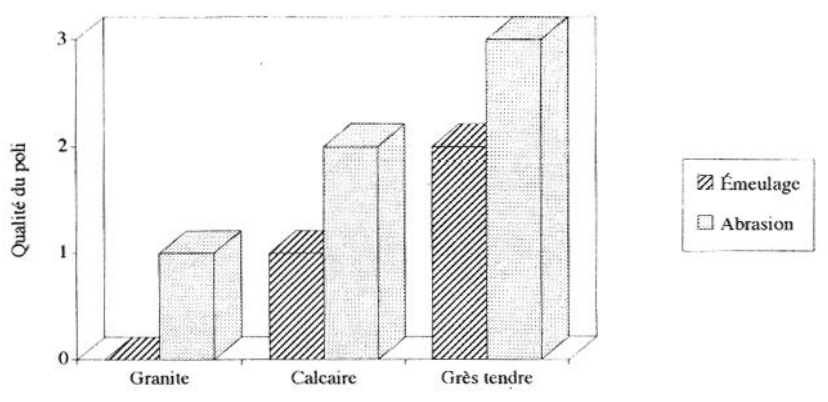

Graphique 3.- Degrés de qualité du poli obtenu expérimentalement lors de l'émeulage et de l'abrasion avec un galet de gneiss peu abrasif $(0$, piètre ; 1 , moyen ; 2 , bon ; 3 , excellent).

à la suite de A. Laming-Emperaire, en trois degrés de finesse différents, du plus grossier au plus fin ${ }^{(16)}$ (1979, p. 49). L'émeulage effectué avec une roche pas ou peu abrasive comme le gneiss permet d'obtenir une régularisation de la surface, plus ou moins rapidement et efficacement selon la dureté des matériaux et leur qualité abrasive. Il est totalement inefficace sur le granite mais l'est davantage sur le

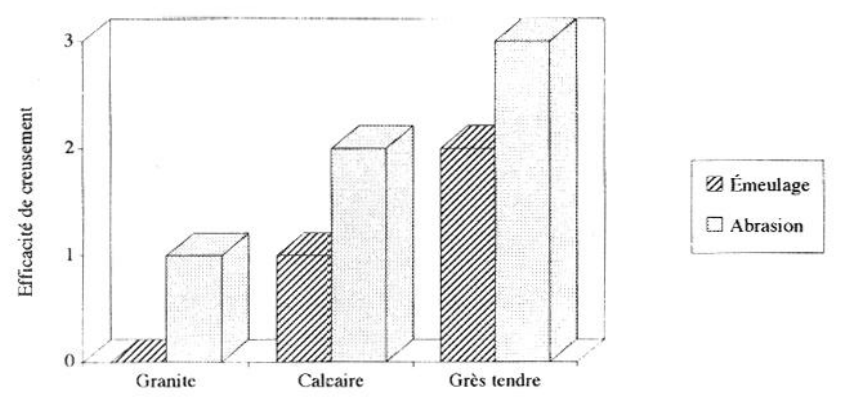

Graphique 4.- Degrés d'efficacité de creusement de la cuvette lors de l'émeulage et de l'abrasion avec un galet de gneiss peu abrasif $(0$, inefficace ; 1 , peu efficace ; 2 , efficace ; 3 , très efficace).

grès : c'est alors la roche travaillée elle-même qui, en se "décomposant", libère des éléments abrasifs. Pour les trois matières premières testées, l'abrasion est préférable à l'émeulage. Le recours à un élément abrasif intermédiaire n'est pas nécessaire lors de l'utilisation d'une roche abrasive par elle-même telle que le grès. Que l'élément abrasif soit ajouté ou non, on obtient dans les deux cas une surface lisse, régularisée, plus ou moins concave selon la dureté du matériau. Cette technique paraît la mieux adaptée aux roches tendres comme certains grès, la stéatite et plusieurs roches volcaniques (lave bulleuse par exemple), où elle peut être associée à d'autres techniques telles que le raclage ${ }^{(1)}$.

Ces expérimentations confirment les intuitions suggérées par le matériel archéologique, à savoir qu'à une matière première donnée, on peut associer une technique de façonnage privilégiée. Nous avons comparé l'efficacité de ces techniques pour attaquer différentes matières premières, soit afin de les creuser, soit afin de les aplanir. Comme pour les degrés de qualité du poli obtenu, des degrés d'efficacité ont été délimités, du plus inefficace au plus efficace (graph. 5). Les Paléolithiques n'ignoraient donc rien des caractéristiques physiques des matériaux qu'ils avaient à leur disposition et les exploitaient au mieux. Ceci étant, nous avons vu que le façonnage de ces matériaux souvent ingrats à travailler est assez rare et que l'homme préférait sélectionner les galets, blocs ou plaquettes dont la

\footnotetext{
16. Comme elle, nous ne proposons aucune mesure permettant de passer d'un scuil de finesse à un autre, chaque chercheur devant établir son propre code après examen des pièces à la loupe binoculaire.

17. Que cette roche doive être travailléc en percussion posée sous peine de se briser est confimée par nos expérimentations en cours sur la stéatite, et par des informations ethnographiques. Ainsi, d'après Moussa Albaka, forgeron touareg nigérien, les artisans spécialisés dans la confection d'objets en stéatite régularisent leurs surfaces par abrasion à l'aide de papier de verre, obtenant ainsi une surface rappelant celle du marbre. A notre connaissance, aucune pièce paléolithique en stéatite n'a été régularisće par abrasion et toutes présentent encore des traces de raclage.
} 


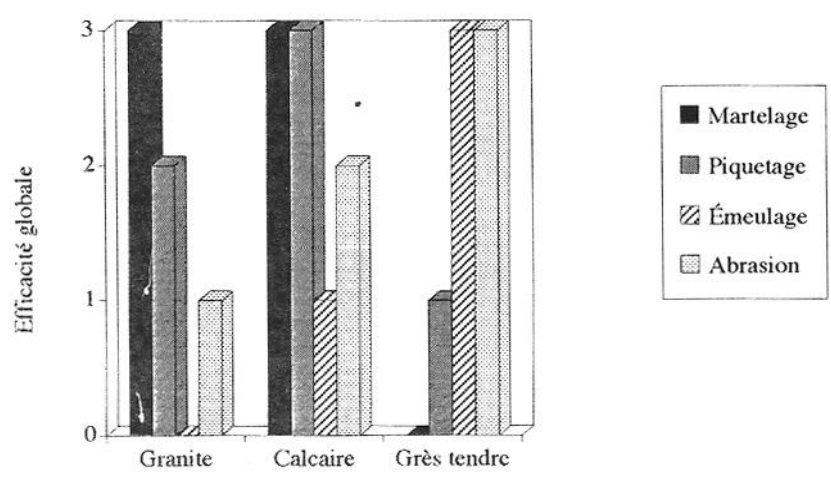

Graphique 5.- Comparaison de l'efficacité globale (creusement et poli) des quatre techniques expérimentées : martelage avec percutcurs de basalte, piquetage avec pics en silex, émeulage et abrasion avec galet de gneiss peu abrasif $(0$, inefficace ; 1 , peu efficace ; 2 , efficace ; 3 , très efficace).

forme naturelle pouvait être exploitée telle quelle. Constatant la grande variabilité pétrographique des meules préhistoriques, A. Schoumacker s'est interrogée sur leurs qualités abrasives. A partir de données pétrographiques concernant les meules modernes, elle parvient a la conclusion que les hommes préhistoriques n'ont pas pu ignorer les qualités abrasives propres à chaque roche. Elle en conclut - et c'est là que nos démarches se rencontrent - que "la découverte sur un même site archéologique d'un grès fin et d'un fragment de granite aura des conséquences importantes sur l'interprétation fonctionnelle" (Schoumacker 1993, p. 170). Cela confirme que les classifications typologiques et fonctionnelles de ce type d'outillage doivent tenir compte de ce paramètre important qu'est la matière première.

Il semble bien que les différentes techniques de façonnage de la pierre (autres que la taille) aient toutes été inventécs au début du Palćolithique supérieur, ou même avant pour certaines d'entre clles ${ }^{(18)}$. Dès l'Aurignacien, ces techniques attcignent un haut degré de perfection et sont appliquées non seulement à la pierre mais aussi aux matières dures animales. Mais ces gestes techniques qui existaient peut-être déjà antéricurement restent peu répandus et ce n'est qu'au Gravettien et surtout au Magdalénien qu'ils deviennent plus systématiques. Les premiers Homo sapiens sapiens scmblent donc avoir très tôt compris et exploité les modes d'action sur la matière et les avoir adaptés aux matières animales et minérales qu'ils avaient à leur disposition. Notons toutefois que les pierres façonnées et brisćes au cours de leur fabrication ne sont pas rares dans les sites archéologiques (de Beaune 1987, p. 68). Nous avons constaté expérimentalement qu'il suffit d'un seul impact un peu trop énergique pour briser une pièce en calcaire, même assez épaisse, et réduire ainsi à néant un travail de plusieurs heures (ibid., p. 104). Les deux fragments obtenus ainsi bien involontairement étaient assez semblables à nombre d'exemplaires paléolithiques.

Ces expérimentations soulèvent des questions que de nouvelles expérimentations ainsi que des observations macro- et microscopiques permettront peut-être d'approfondir. On peut se demander en particulier avec quels outils ces roches ont été façonnées. Peut-on trouver et identifier, sur un site, des pierres façonnées et les outils ayant servi à leur façonnage ? La réponse ne peut se faire qu'au cas par cas. En théorie, on ne peut retrouver les outils ayant scrvi au façonnage de ces objets que s'ils ont servi sur le licu même de leur fabrication ou bien s'ils ont été cassés en cours de fabrication. A Solvieux, deux lampes de grès entièrement façonnées ont été retrouvées a proximité de ciseaux de diabase qui auraient pu servir, d'après C. Singer, à leur confection. J. Sackett, qui a examiné leur émoussé au microscope, n'a cependant pas relevé de traces de la roche abraséc permettant d'associer avec certitude les lampes aux ciseaux mais cette absence peut être due a l'altération dans le sol, ou au lavage qu'ont subi les ciscaux (de Bcaune, Roussot, Sackett 1986, p. 111112). Sur le site magdalénien final du Rocher de la Caillc, dc nombreux petits galets de stéatite creusés par raclage et parfois ornés de décors sculptés ou gravés sont en cours d'étude. Les expérimentations que nous menons en collaboration avec Ph. Alix permettront pcut-être de mettre en relation l'industrie lithique retrouvée avec la fabrication de ces petits récipients. Si l'expérimentation et l'observation microscopique de l'outillage archéologique permettent de conclure que ces outils ont bien servi à la confection des récipients en stéatite, on pourra alors conclure que ces derniers ont bien été retrouvés sur le licu de leur fabrication. Le fait qu'ils soient tous brisés pourrait confirmer que nous avons affaire à une "fabrique" de récipients en stéatite et que seuls sont restés sur le site les exemplaires cassés.

On voit donc que ce type d'étude peut permettre de mettre en évidence la présence d'activités spécialisées, et, partant, de préciser la fonction même d'un site. Par ailleurs, il permet d'interpréter certains comportements paléolithiques puisqu'on peut en conclure à un traitement différencié des roches au Paléolithique supérieur et donc à une gestion particulière des matières premières disponibles. 


\section{BIBLIOGRAPHIE}

BEAUNE, S. A. de, 1987. Lampes et godets au Paléolithique. XXIIİ̀me suppl. à Gallia Préhistoire. Paris : Éd. du CNRS, 280 p., 93 fig., 81 tabl., 16 pl. h.-t.

BEAUNE, S. A. de, 1989 a. Essai d'une classification typologique des galets et plaquettes utilisés au Palcolithique. Gallia Préhistoire, vol. 31, p. 27-64, 14 fig., 3 tabl.

BEAUNE, S. A. de, 1989 b. Fonction et décor de certains ustensiles palcolithiques en pierre. L'Anthropologie, 1989, vol. 93, n² 2, p. 547-584.

BEAUNE, S. A. de, 1992. Une lacune à combler : l'étude des galets utilisés au Palćolithique. Les nouvelles de l'archéologie, $\mathrm{n}^{\circ} 46, \mathrm{p}$. 48-52, 2 tabl.

BEAUNE, S. A. de, 1993. Nonflint Stone Tools of the Early Upper Palcolithic. In : Before Lascaux : Complex record of the Early Upper Paleolithic. Ed. by H. Knecht, A. Pike Tay and R. White, Boca Raton, Florida : CRC Press Inc., chapt. 11, p. 163191, 4 fig., 4 tabl.

BEAUNE, S. A. de, à paraître. Utilisation des galets au Paléolithique. Suppl. à Gallia Préhistoire. Paris : CNRS-Éditions.

BEAUNE, S. A. de, BUISSON, D., à paraître. Différenciation spatio-chronologique de l'utilisation des galets au cours du Palcolithique supéricur européen : les cas d'Isturitz (Pyrénées-Allantiques) ct de La Vache (Ariège). Actes du 118ème Congrès national des Sociétés historiques et scientifiques, Pau (25-29 octobre 1993). Paris : Ed. du Comité des Travaux Historiques et Scientifiques.

BEAUNE, S. A. de, ROUSSOT, A., SACKFTT, J., 1986. Les lampes de Solvicux (Dordogne). L'Anthropologie, tome 90, $\mathrm{n}^{\circ}$ 1, p. 107-119, 8 fig.

CHEVALIER, J., INIZAN, M.-L., TIXIER, J., 1982. Une technique de perforation par percussion de perles en cornaline (Larsa, Iraq). Paléorient, vol. 8/2, p. 55-65, 7 fig.

DELLUC, B. et G., 1978. Les manifestations graphiques aurignaciennes sur support rocheux des environs des Eyzies (Dordogne). Gallia Préhistoire, T. 21, p. 213-438, 96 fig.
GARANGER, J., 1968. Herminettes océaniennes, éléments de typologie. 29 p., 15 fig., bibl., mim.

LAMING-EMPERAIRE, A., 1979. L'analyse descriptive de l'industrie lithique. In : Enregistrement et analyse de données archéologiques. Essai sur l'analyse descriptive d'industries lithiques américaines. Textes réunis et présentés par P. Plumet. Montréal : Paléo-Québec 9, p. 9-58, 13 fig.

LEROI-GOURHAN, A., 1943. L'homme et la matière. Évolution et techniques. I. Paris : Albin Michel, 348 p., 577 fig.

MACRAE, R.J., MOLONEY, N. (cd.), 1988. NonFlint Stone Tools and the Paleolithic Occupation of Britain. Oxford : BAR British Scries 189, 263 p., fig., tabl.

MADSEN, B., 1984. Flint axe manufacture in the Neolithic : experiments with grinding and polishing of thin-butted flint axes. Journal of Danish Archaeology, vol. 3, p. 47-62, 14 fig.

MCARTHY, F.D., 1976. Australian aboriginal stone implements. Sydney: The Australian Muscum Trust, $2 \mathrm{~d}$ ed., 108 p., 72 fig.

PROUS, A., 1977. Les sculptures zoomorphes du Sud brésilien et de l'Uruguay. Paris : Cahiers d'Archéologie d'Amérique du Sud, 5, 178 p., tabl., 7 pl.

RICQ-DE BOUARD, M., 1983. Les outils lithiques polis du Sud de la France. Paris : Éd. du CNRS, 37 p., 6 fig., tabl.

ROODENBERG, J.J., 1983. Traces d'utilisation sur les haches polies de Bouqras (Syrie). In : Traces d'utilisation sur les outils néolithiques du ProcheOrient. Table ronde CNRS à Lyon (8-10 juin 1982), Lyon : GIS, Maison de L'Orient, p. 177-188, 4 fig., 1 pl.

ROSTAIN, S., WACK, Y., 1987. Haches et herminettes en pierre de Guyane française. Journal de la Société des Américanistes, tome LXXIII, p. 107-138, 11 fig.

SCHOUMACKER, A., 1993. Apports de la technologie et de la pétrographie pour la caractérisation des meules. In : Traces et fonction : les gestes retrouvés. Colloque international de Liège (8-10 décembre 1990), Liège : Éd. ERAUL, vol. 50, p. 165-176, 4 fig., tabl. 
SUSSMAN, C., 1988. A microscopic analysis of usewear and polish formation on experimental quartz tools. Oxford : BAR International Series 395, 205 p., 39 fig., 58 pl., tabl.

WILLOUGHBY, P.R., 1987. Spheroids and Battered Stones in the African Early and Middle Stone Age. Oxford : BAR International Series 321, 254 p., fig., tabl. 


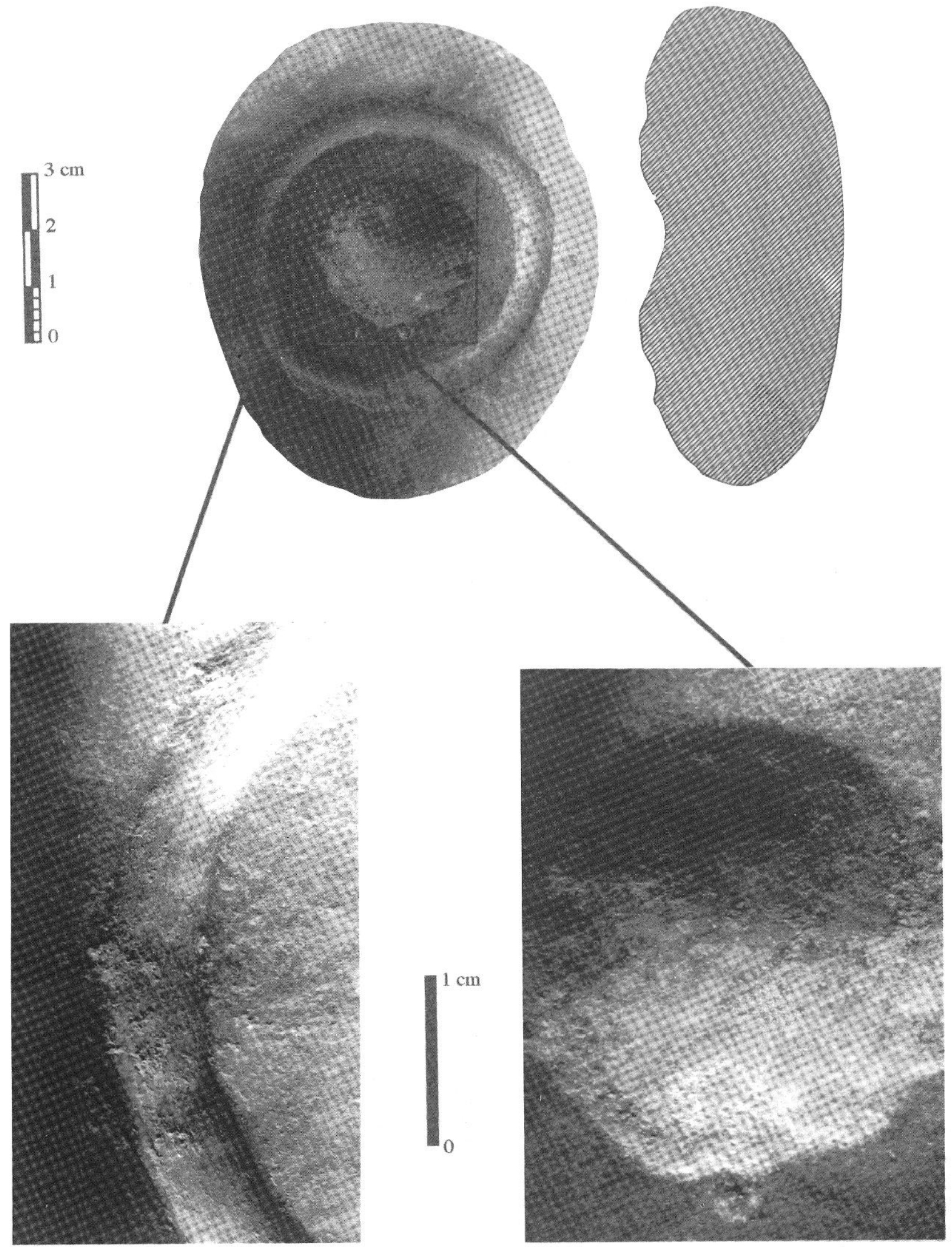

Figure 1.- Galet de quartz brun. Abri de la Garenne, Saint-Marcel (Indre). Magdalénien V ou VI. La cuvette et la cannelure circulaire ont été obtenues par piquetage. Le piquetage de la cannelure est partiellement oblitéré par le polissage de régularisation postérieur. M.A.N., coll. Benoist, $n^{\circ} 46708$. 


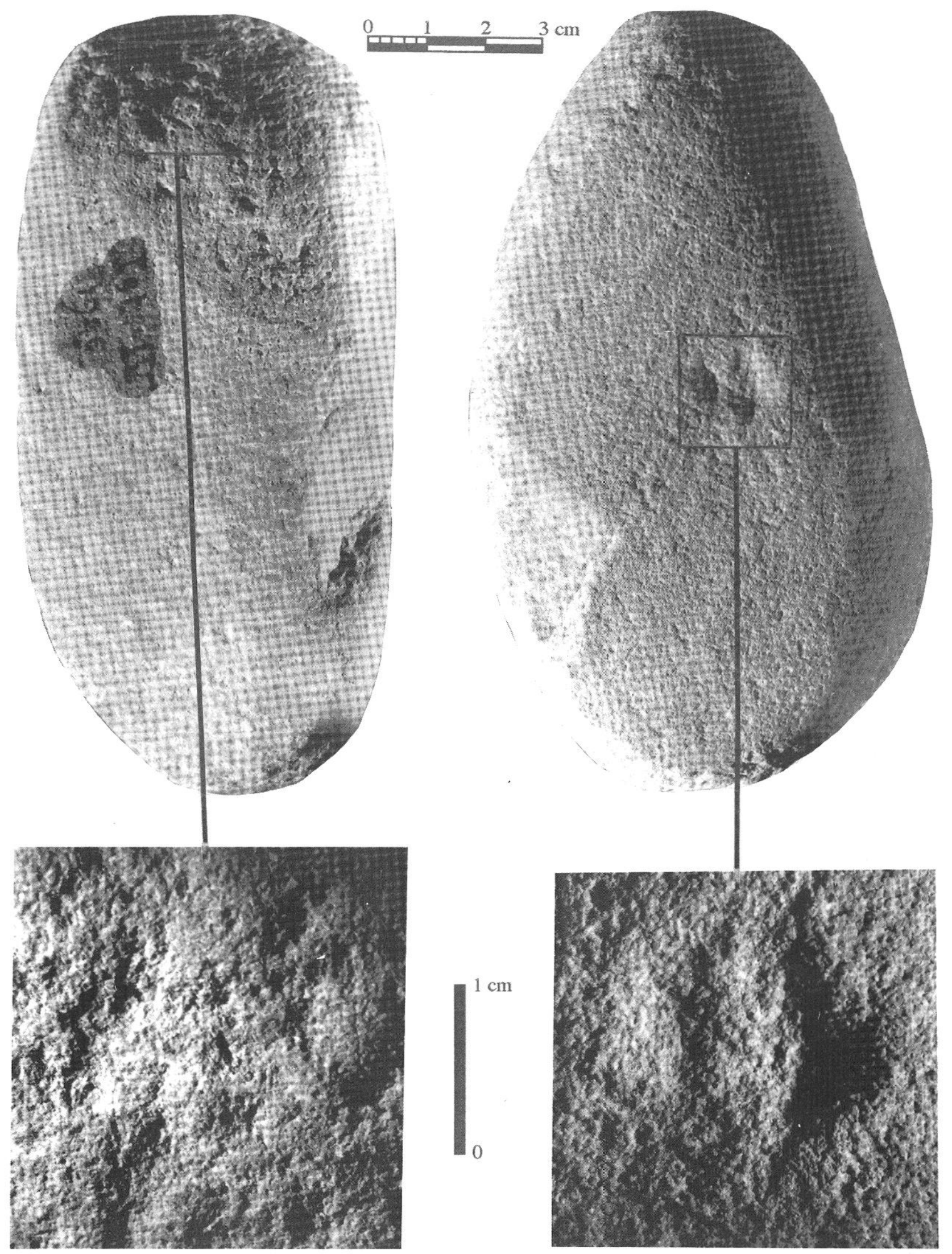

Figure 2.- Broyeur en grès rose à grain fin. Grotte d'Isturitz (Pyrénées-Atlantiques). Périgordien $\mathrm{V}$ à burins de Noailles (niveau IV). Des traces d'impacts recouvrent partiellement le poli d'utilisation des faces et des flancs. Celles qui sont visibles au centre des faces ont pu être produites lors de l'utilisation du galet en marteau ou en enclume ; celles des flancs pourraient avoir été réalisées délibérément, par martelage, afin de redonner sa rugosité première à la surface destinée à moudre ou broyer. M.A.N., coll. Saint-Périer, $n^{\circ} 83889$. 


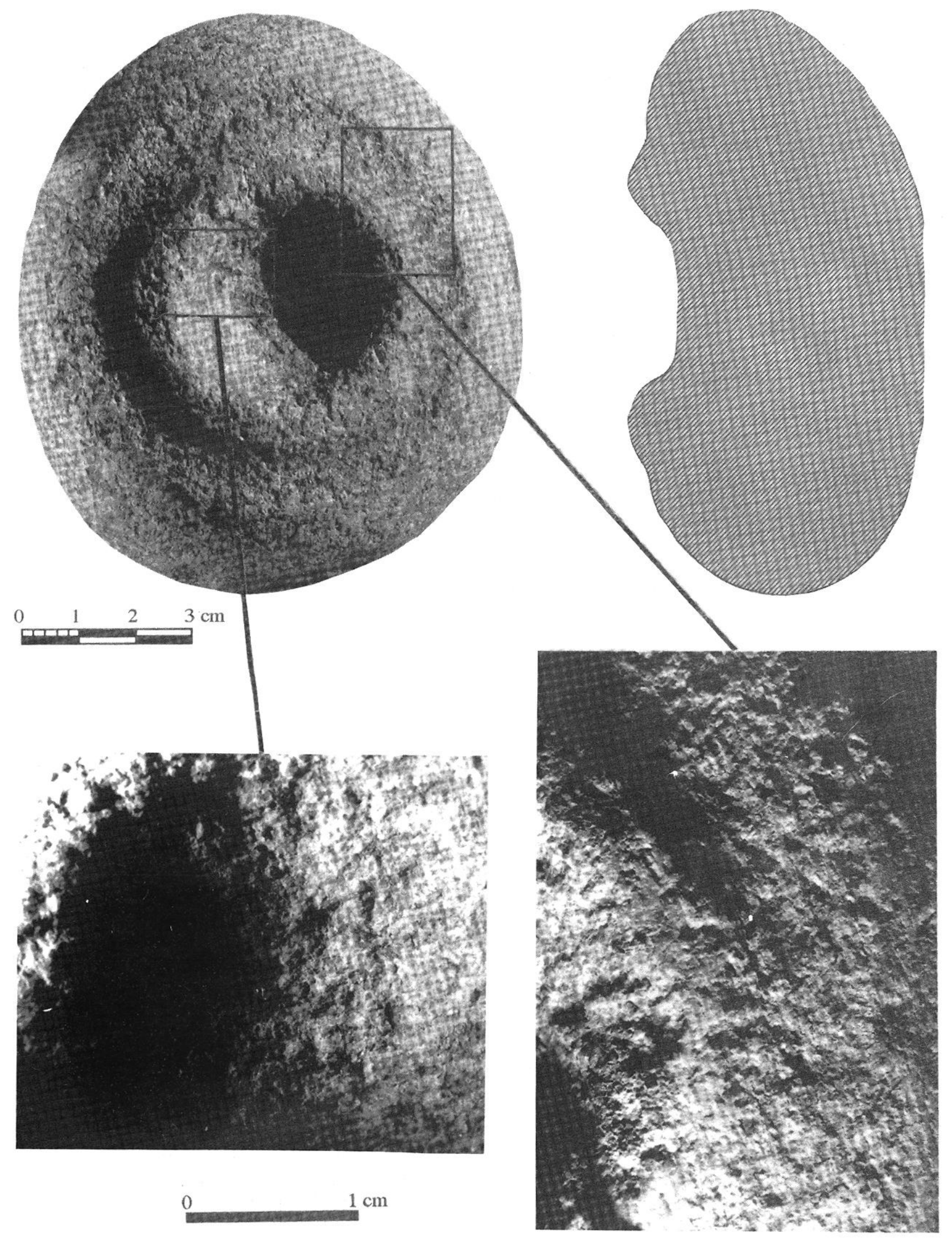

Figure 3.- Godet en calcaire. La Rochette, Saint-Léon-sur-Vézère (Dordogne). Périgordien supérieur. La cuvette bien circulaire a sans doute été creusée par martclage. M.A.N., coll. Daniel, $\mathrm{n}^{\circ} 83467$. 

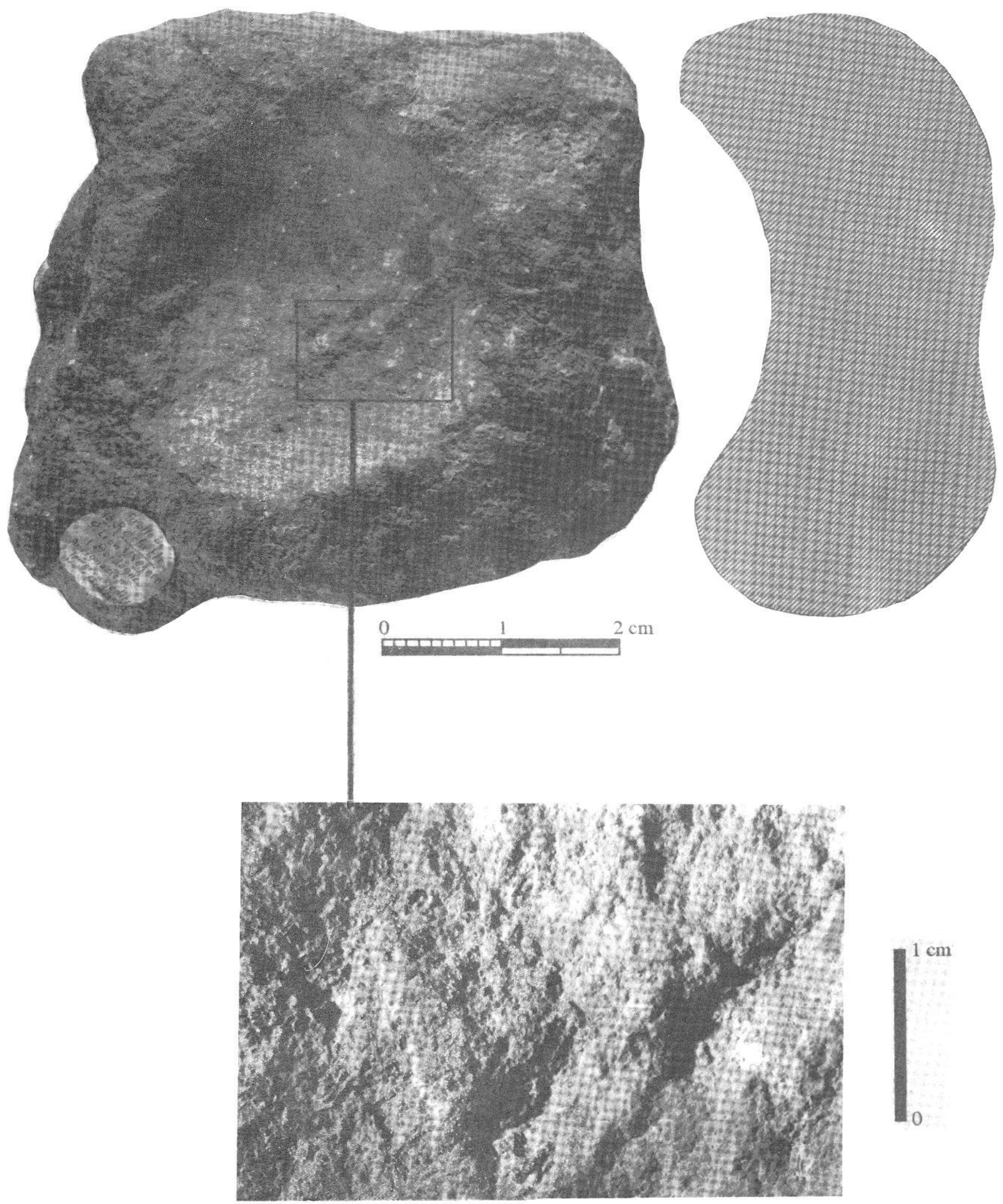

Figure 4.- Galet de granite micacé. Abri classique de Laugerie.Basse (Dordogne). Magdalénien moyen ou supérieur. La cuvette bien circulaire et la cannelure ont sans doute été obtenues par un piquetage assez fin. M.A.N., coll. Girod, $\mathrm{n}^{\circ} 54304$. 


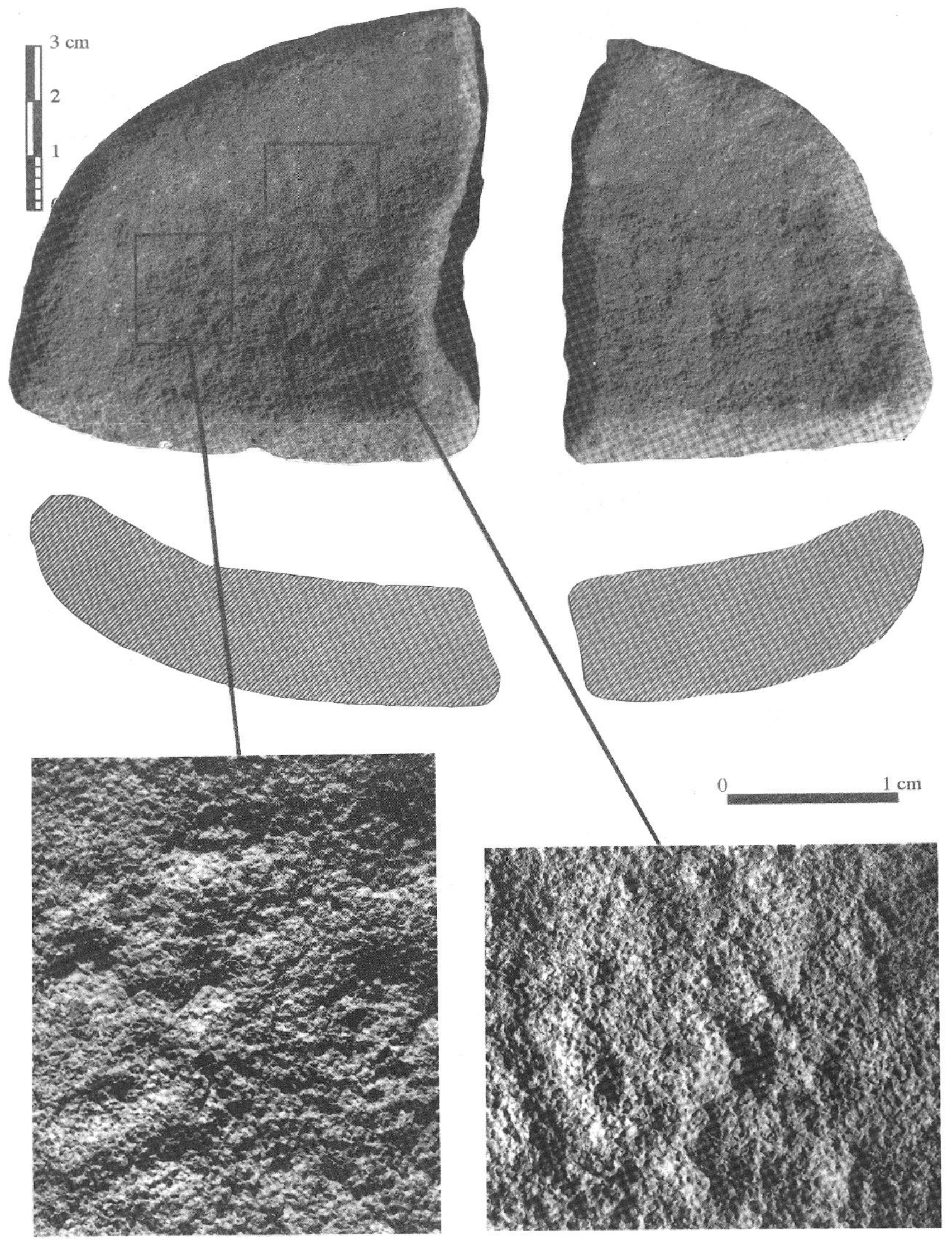

Figure 5.- Lampe en grès gris tendre, un peu micacé. Grotte d'Isturitz. Périgordien V à burins de Noailles (niveau IV). La cuvette a été façonnée par piquetage. On observe sur les versants de la cuvette, quelques traces de dérapage du pic. M.A.N., coll. Saint-Périer, $n^{\circ} 83889$. 


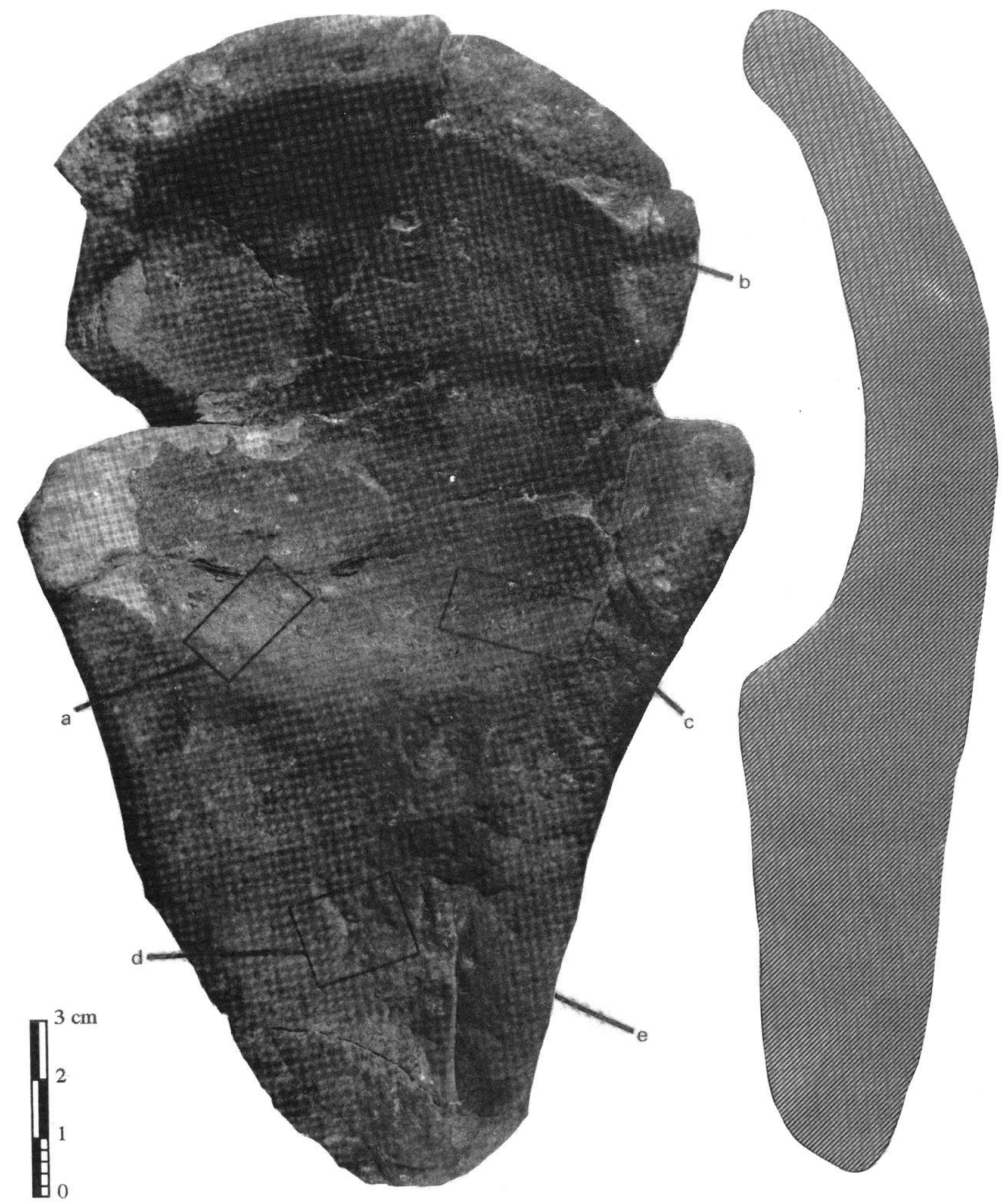

Figure 6.- Lampe en grès rouge tendre à grain très fin légèrement micacé. Grotte des Scilles, Lespugue (Haute-Garonne). Magdalénien III ou IV. Cette lampe a été entièrement sculptée, sans doute par raclage, puis régularisée par polissage (b). Des traces nettes sur le pourtour de la cuvette indiquent que ce travail de régularisation s'est fait en un mouvement circulaire (a et c). Le manche et le revers ont été régularisés par polissage. Quelques traces d'impacts grossiers ont été interprétées par R. de Saint-Périer comme des traits gravés (d). M.A.N., coll. Saint-Périer, $n^{\circ} 86775$. 

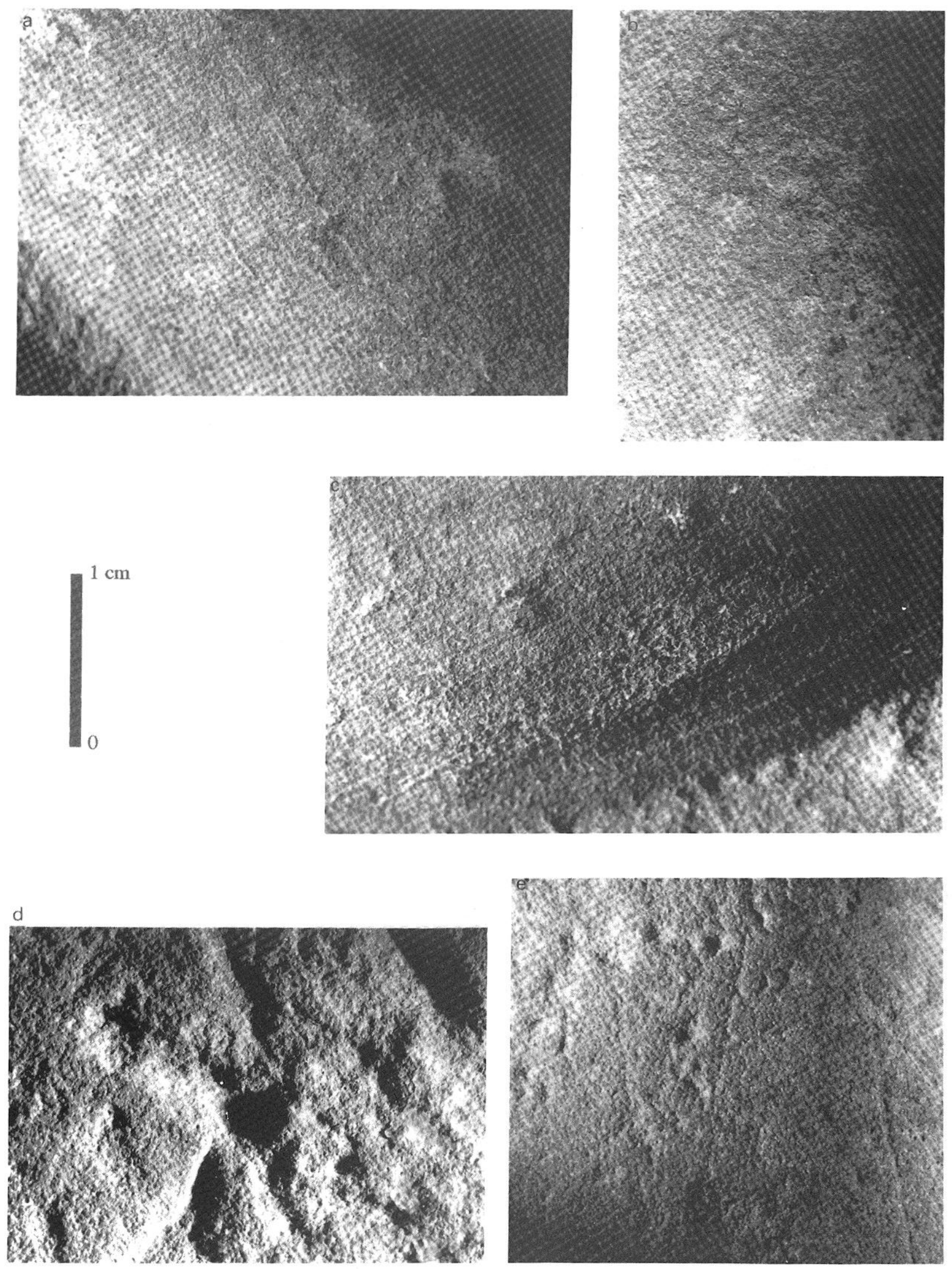

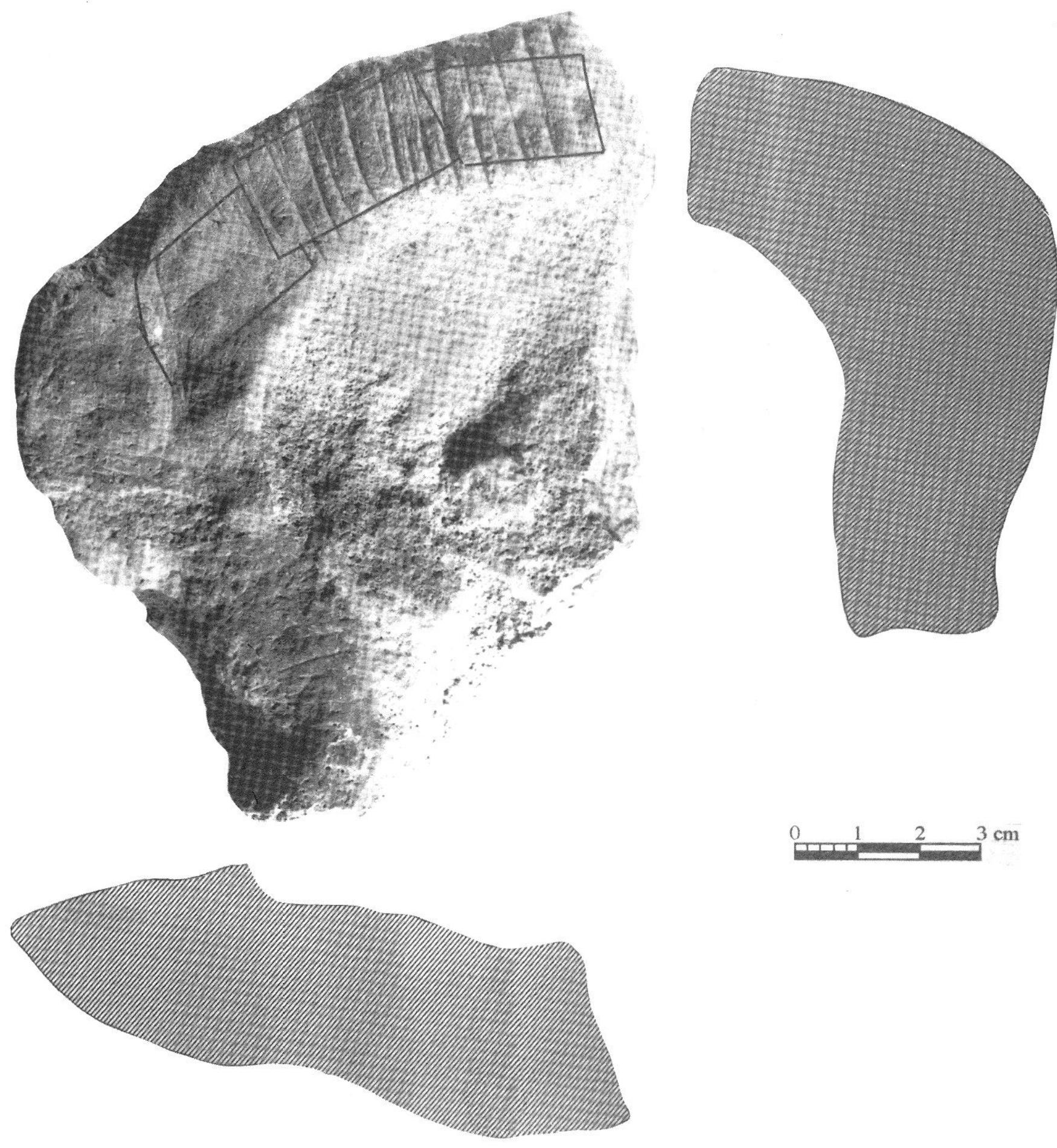

Figure 7.- Fragment de récipient en calcairc. Grotte de La Marche, Lussac-les-Châteaux (Vienne). Magdalénien moyen ou supérieur. La cuvette, naturelle, est peut-être une empreinte de fossile. La margelle a été légèrement régularisée par raclage puis gravée d'une série de traits parallèles. M.A.N., coll. Fitte, sans numćro. 


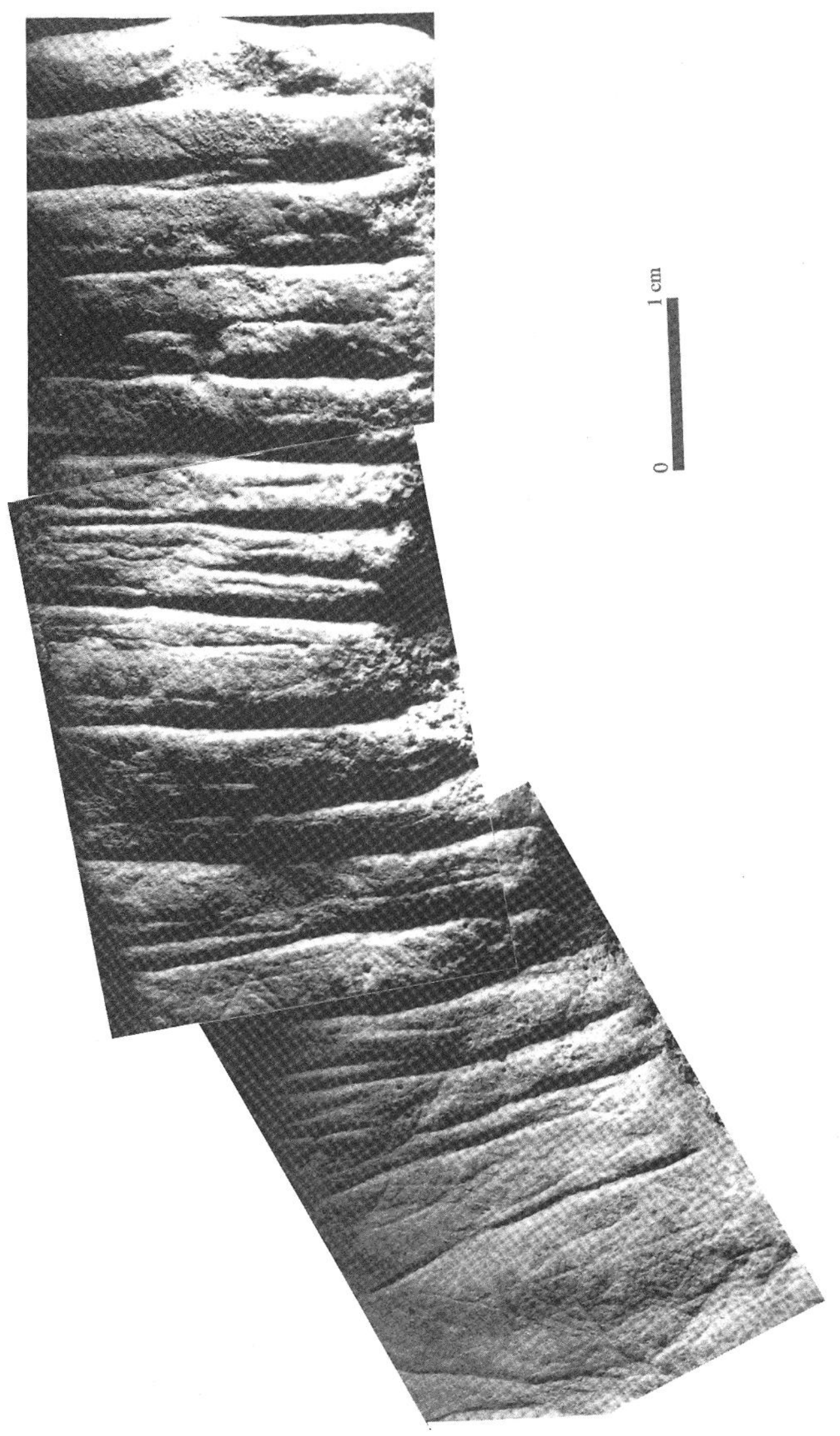



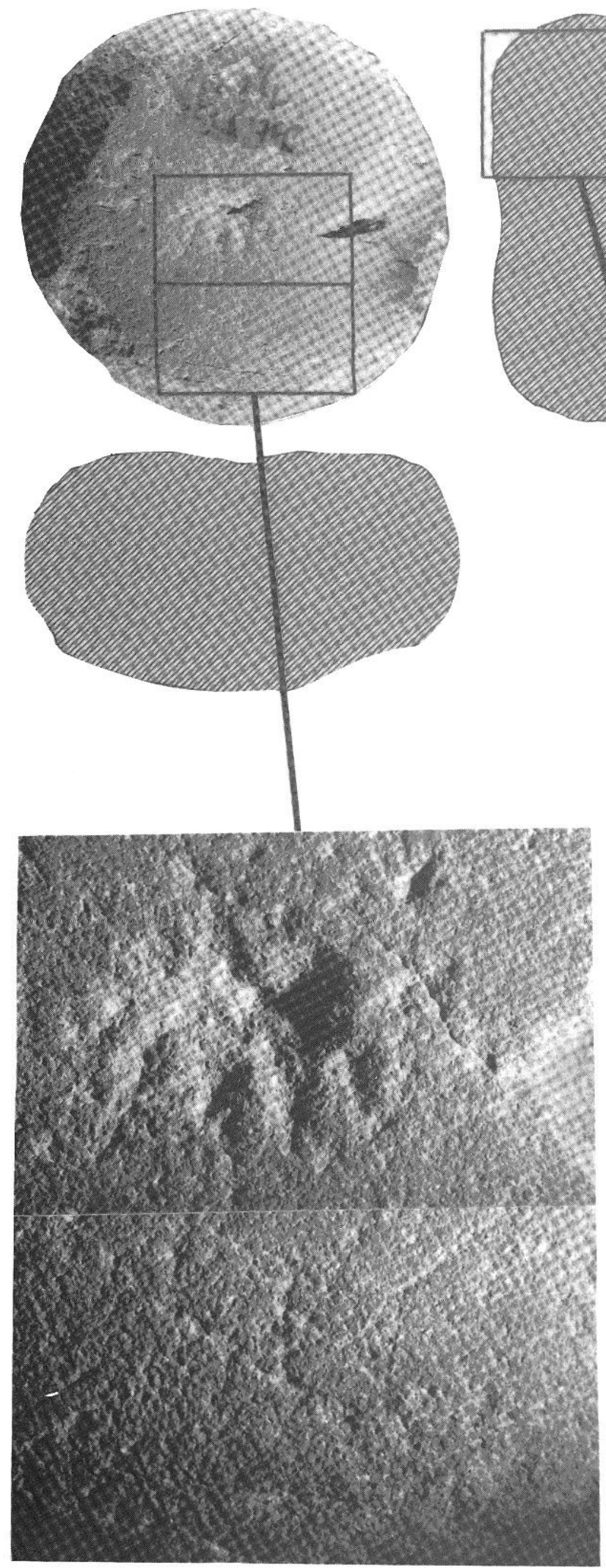
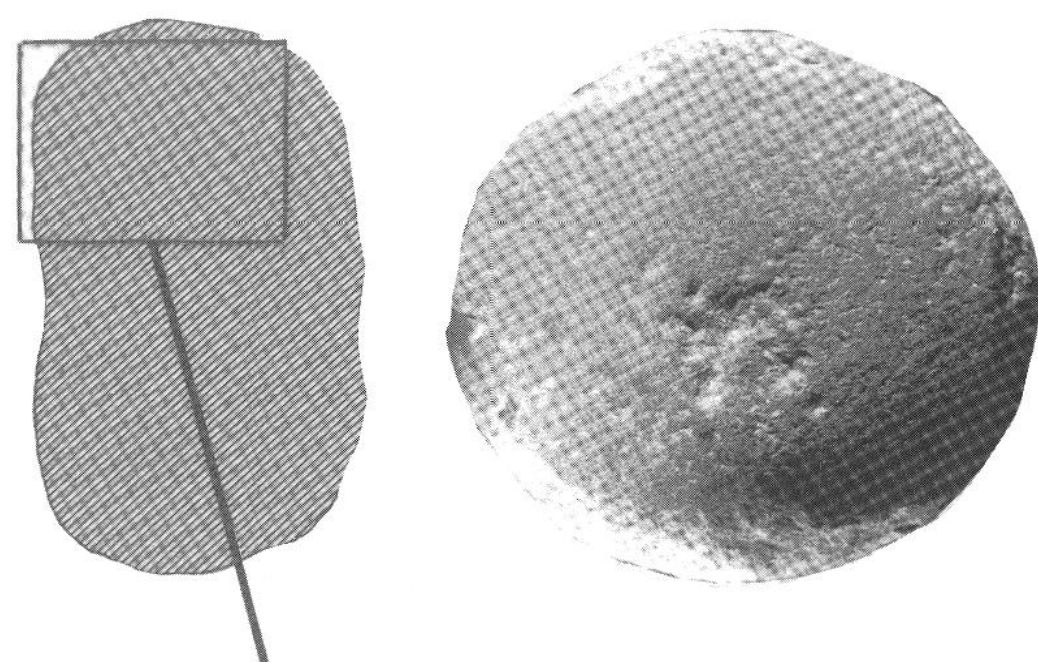

\begin{tabular}{llll}
0 & 1 & 2 & 3 \\
\hline
\end{tabular}
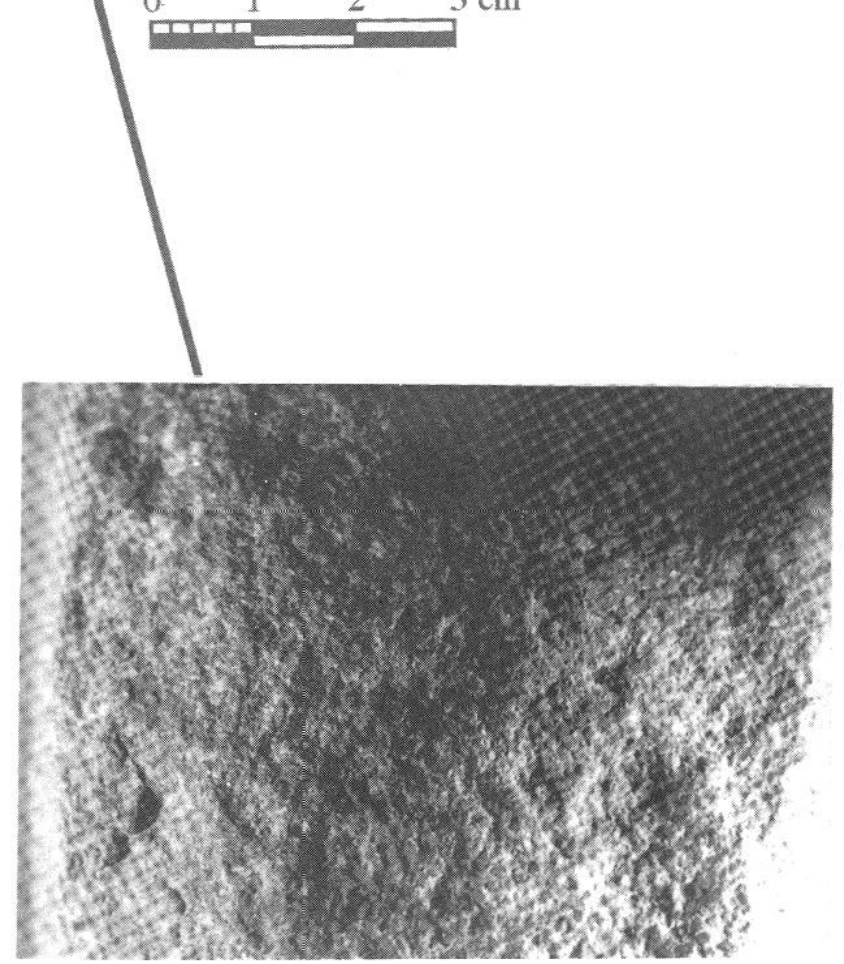

0 $1 \mathrm{~cm}$

Figure 8.- Petite molette de grès rose à grain fin. Grotte d'Isturitz. Magdalénien moyen (niveau SI). Voir dans le texte pour l'interprétation des traces. M.A.N., coll. Saint-Périer, $n^{\circ} 83892$. 

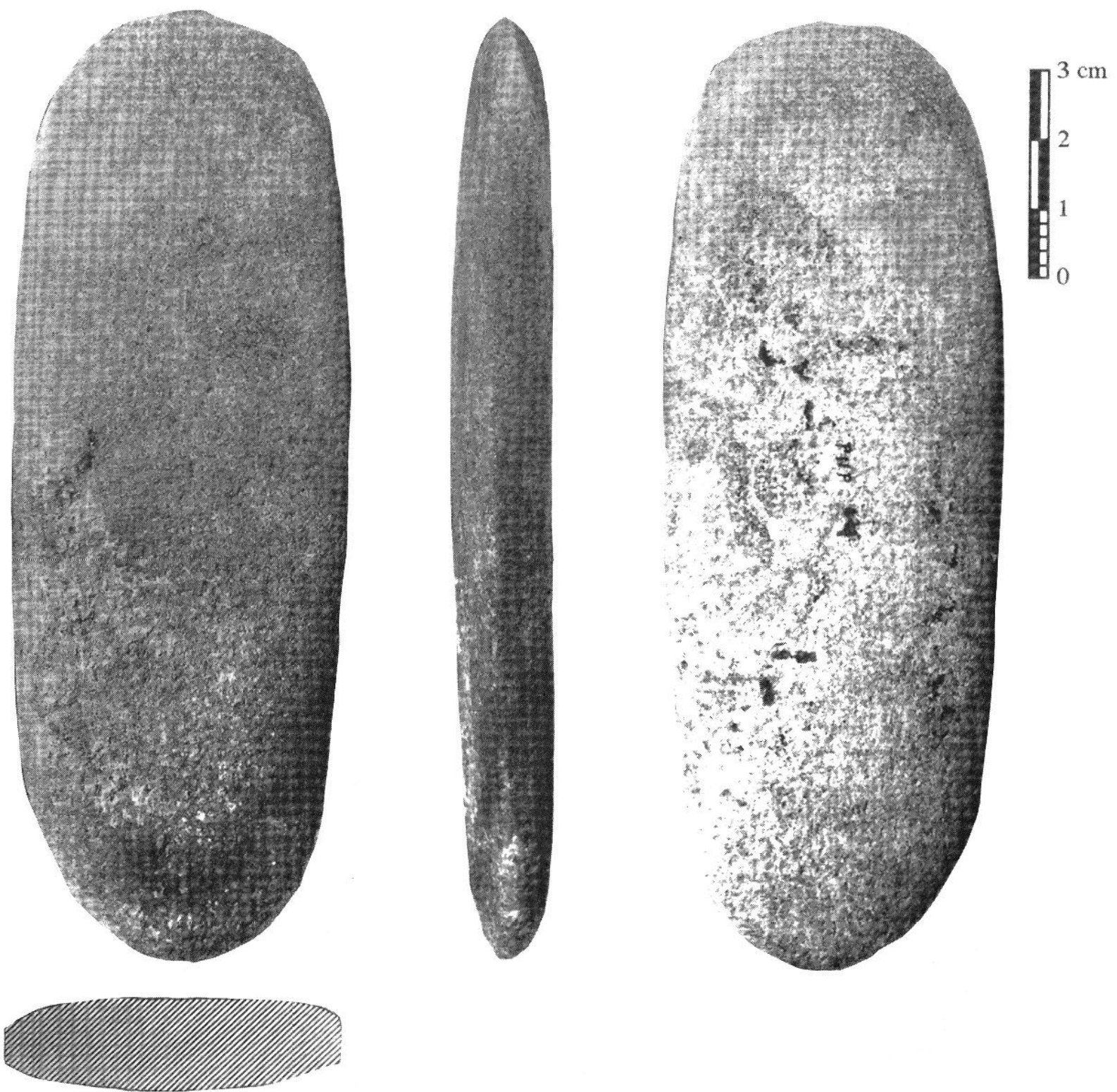

Figure 9.- Galet de quartzite rosé. Pair-non-Pair, Marcamps (Gironde). Aurignacien ou Périgordien supérieur. La totalité des surfaces a été polie. Voir texte et note 9. Musée d'Aquitaine, coll. Daleau. 


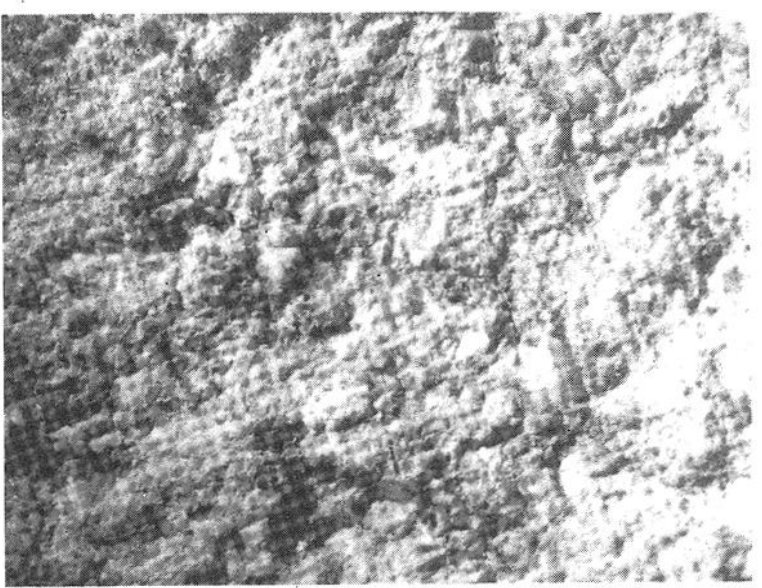

1

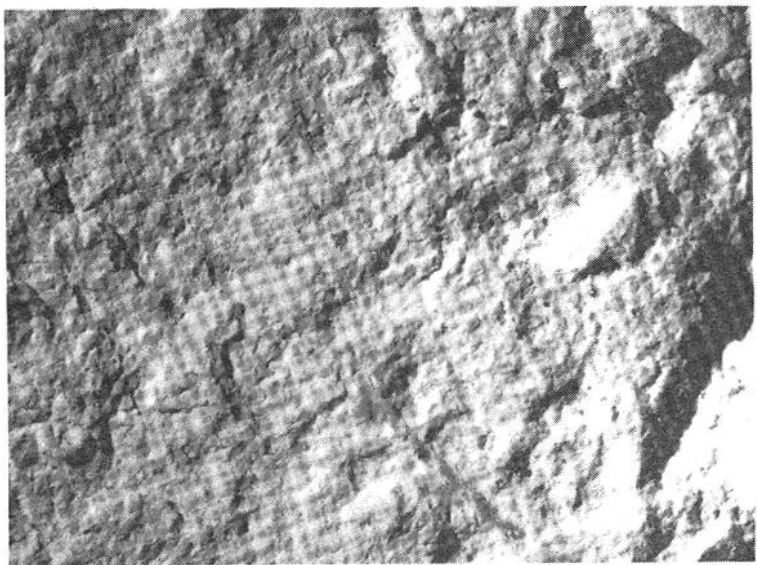

2

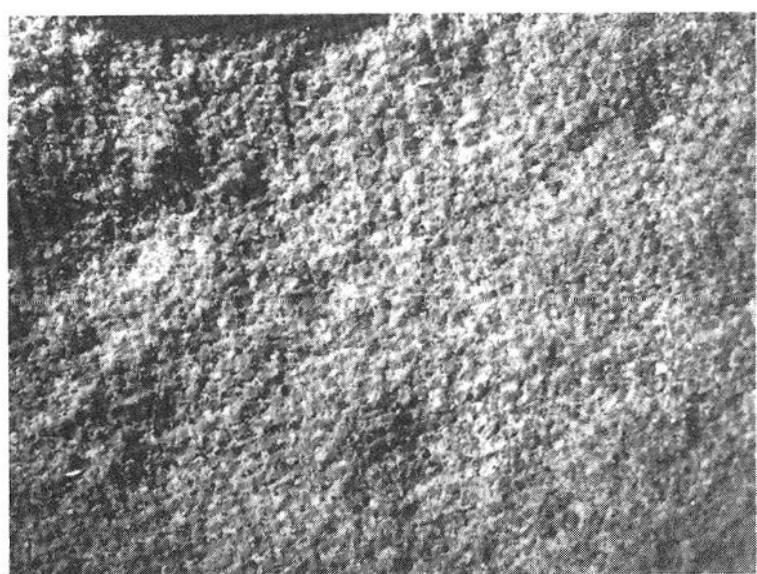

3

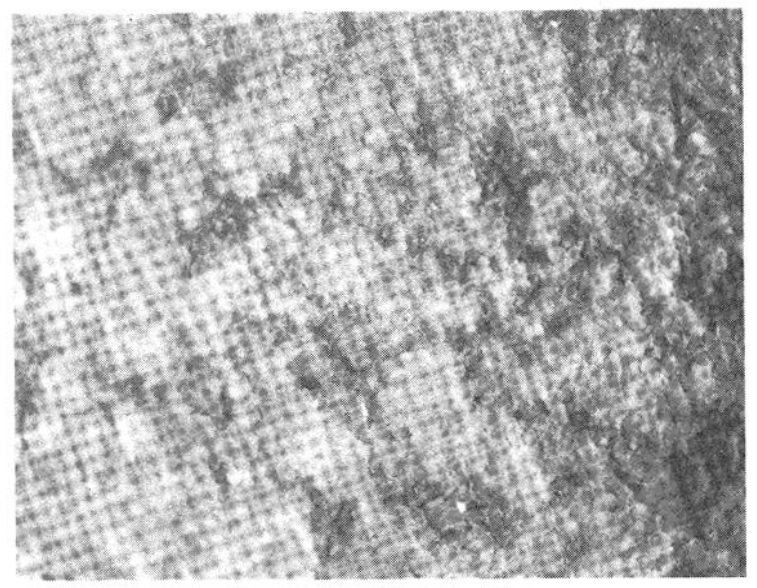

4

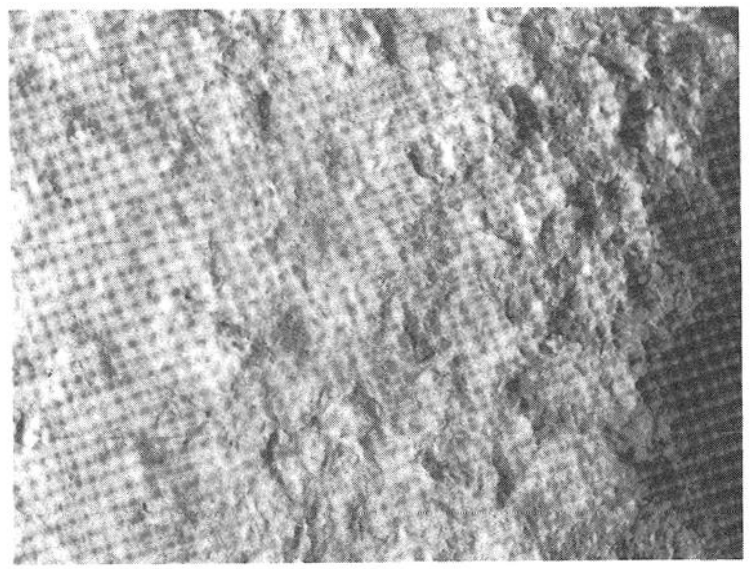

5

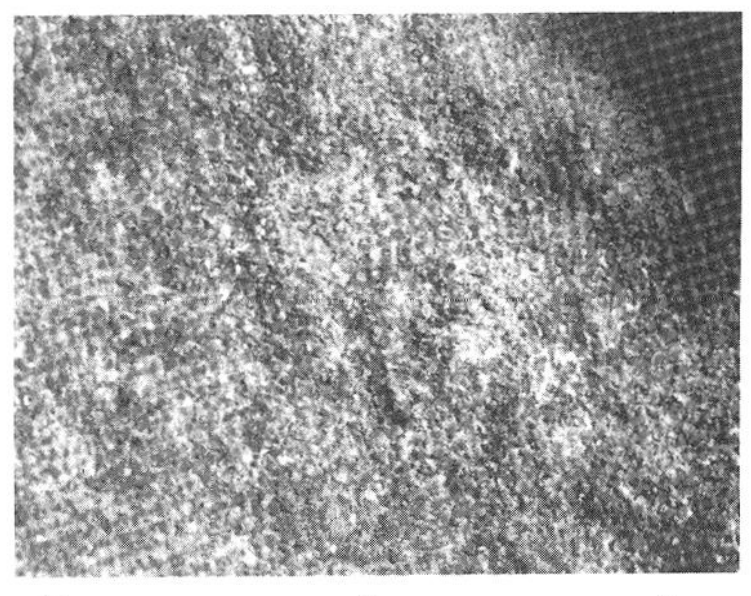
$1 \mathrm{~cm}$

Figure 10.- Traces de façonnage obtenues expérimentalement. Percussion lancée. 1 à 3 , martelage (avec percuteurs de basalte), 1, sur le granite ; 2 , sur le calcaire ; 3 , sur le grès tendre. 4 à 6 , piquetage (avec pics en silex), 4 , sur le granite ; 5 , sur le calcaire ; 6 , sur le grès tendre. 


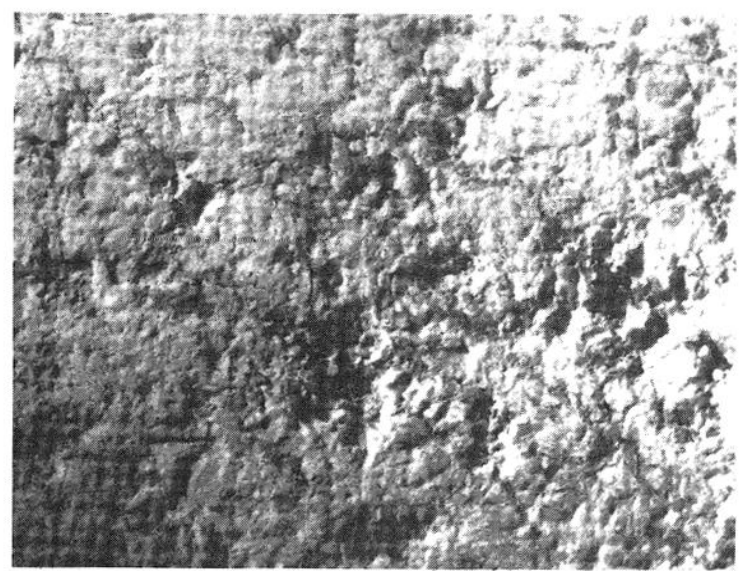

1

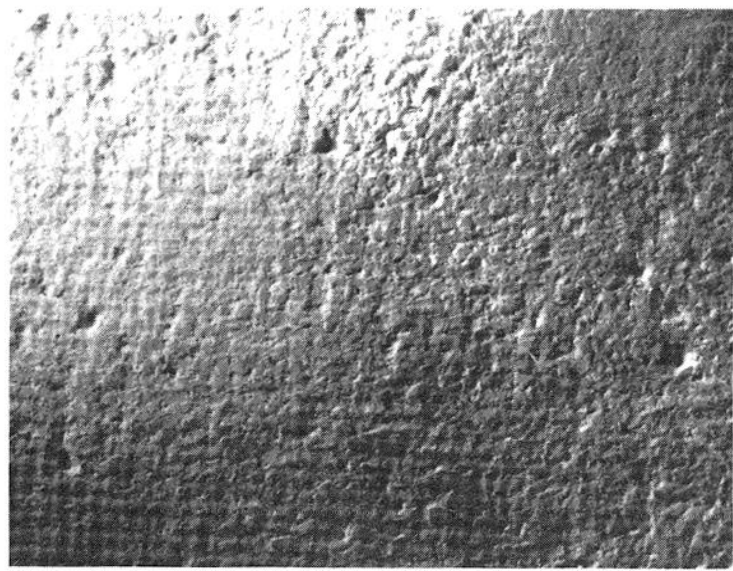

2

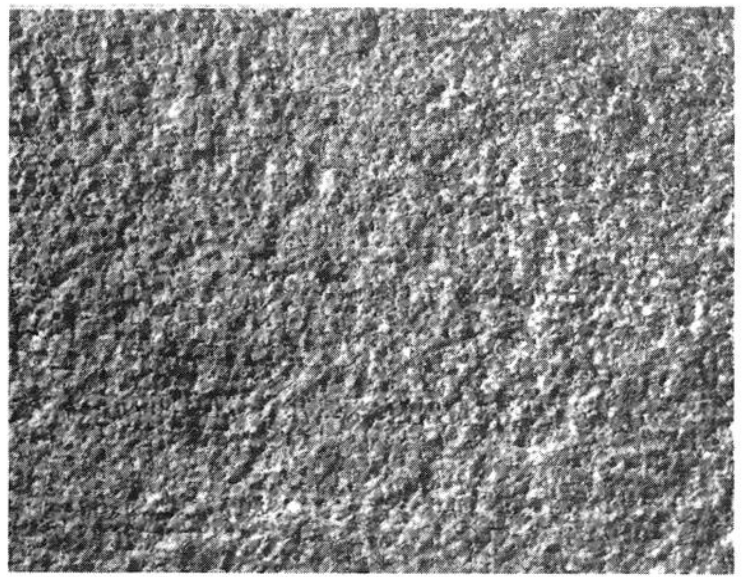

3

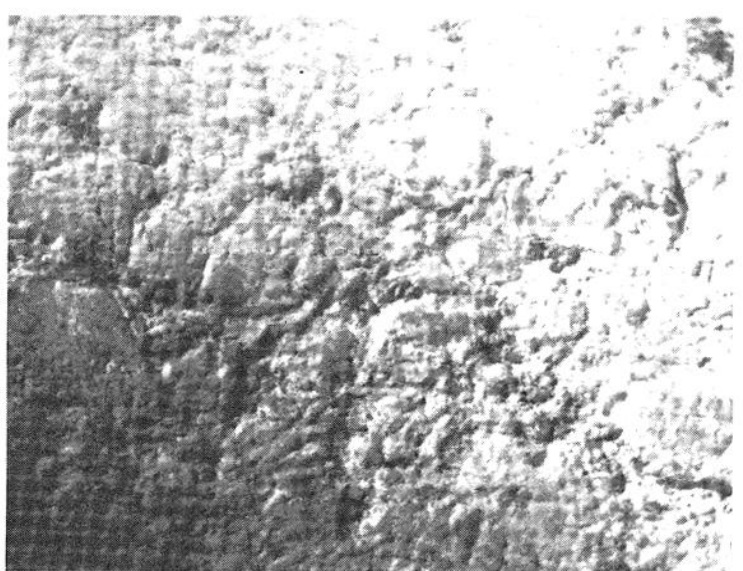

4

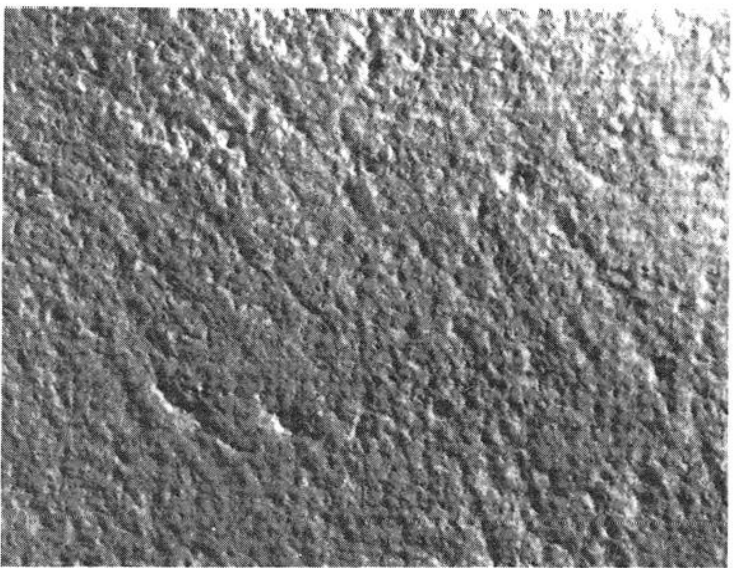

5

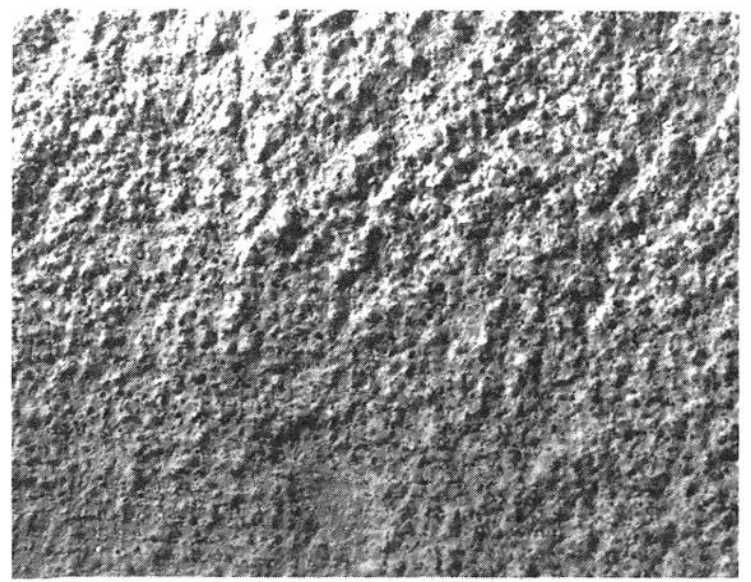

6

$1 \mathrm{~cm}$

Figure 11.- Traces de façonnage obtenues expérimentalement. Percussion posée (avec galet de gneiss peu abrasif). 1 à 3, émeulage, 1 , sur le granite ; 2 , sur le calcaire ; 3 , sur le grès tendre. 4 à 6 , abrasion (avec sable mouillé intermédiaire), 4 , sur le granite; 5 , sur le calcaire $; 6$, sur le grès tendre. 